\title{
EUSO-TA - First results from a ground-based EUSO telescope
}

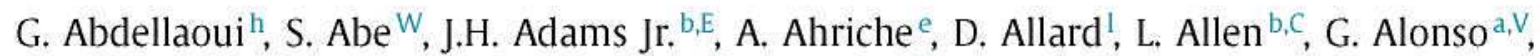
L. Anchordoqui ${ }^{\mathrm{b}, \mathrm{G}}, \mathrm{A}$. Anzalone ${ }^{\mathrm{y}, \mathrm{u}}, \mathrm{Y}$. Arai ${ }^{\mathrm{Y}}, \mathrm{K}$. Asano ${ }^{\mathrm{K}}$, R. Attallah ${ }^{\mathrm{c}}, \mathrm{H}$. Attoui ${ }^{\mathrm{a}}$, M. Ave Pernas $^{\mathrm{a}, \mathrm{U}}, \mathrm{S}$. Bacholle ${ }^{\mathrm{b}, \mathrm{D}}, \mathrm{M}$. Bakiri ${ }^{\mathrm{a}}$, P. Baragatti ${ }^{\mathrm{E}, \mathrm{F}}, \mathrm{P}$. Barrillon ${ }^{\mathrm{j}}, \mathrm{S}$. Bartocci ${ }^{\mathrm{E}}, \mathrm{J}^{\mathrm{C}}$ Bayer ${ }^{\mathrm{q}}$, B. Beldjilali ${ }^{\mathrm{h}}$, T. Belenguer ${ }^{\mathrm{a}, \mathrm{T}}$, N. Belkhalfa ${ }^{\mathrm{a}}$, R. Bellotti ${ }^{\mathrm{r}, \mathrm{s}}$, A. Belov ${ }^{\mathrm{a}, \mathrm{P}}, \mathrm{K}$, Belov $^{\mathrm{b}, \mathrm{F}}, \mathrm{J} . \mathrm{W}$. Belz ${ }^{\mathrm{b} J}$, K. Benmessai ${ }^{a}$, M. Bertaina ${ }^{B, C}$, P.L. Biermann ${ }^{\circ}$, S. Biktemerova ${ }^{a, N}$, F. Bisconti ${ }^{\circ}$, N. Blanc ${ }^{a, Y}$, J. Blecki ${ }^{\mathrm{a}, \mathrm{L}}$, S. Blin-Bondil ${ }^{\mathrm{k}}$, P. Bobik ${ }^{\mathrm{a}, \mathrm{Q}}$, M. Bogomilov ${ }^{\mathrm{i}}$, E. Bozzo ${ }^{\mathrm{a}, \mathrm{Z}}, \mathrm{A}$. Bruno ${ }^{\mathrm{s}}$, K.S. Caballero ${ }^{\mathrm{a}, \mathrm{H}}$, F. Cafagna ${ }^{\mathrm{r}}$, D. Campana ${ }^{\mathrm{w}}$, J-N. Capdevielle ${ }^{\mathrm{l}}$, F. Capel ${ }^{\mathrm{a}, \mathrm{X}}$, A. Caramete $^{\mathrm{a}, \mathrm{M}}$, L. Caramete ${ }^{\mathrm{a}, \mathrm{M}}, \mathrm{P}$. Carlson ${ }^{\mathrm{a}, \mathrm{X}}, \mathrm{R}^{\mathrm{C}}$. Caruso ${ }^{\mathrm{t}, \mathrm{u}}, \mathrm{M}$. Casolino ${ }^{\mathrm{Z}, \mathrm{Z}}$, C. Cassardo $^{\mathrm{B}, \mathrm{C}}$, A. Castellina ${ }^{\mathrm{B}, \mathrm{D}}$,

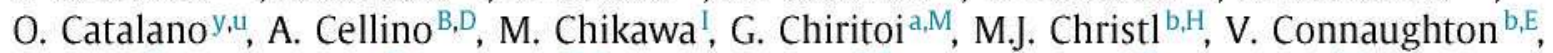

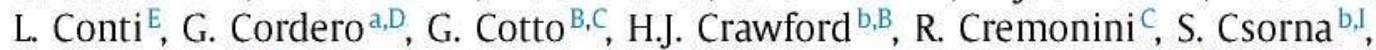

A. Cummings ${ }^{\mathrm{b}, \mathrm{D}}$, S. Dagoret-Campagne, C. De Donato ${ }^{z}$, C. de la Taille ${ }^{\mathrm{k}}$, C. De Santis ${ }^{2}$, L. del

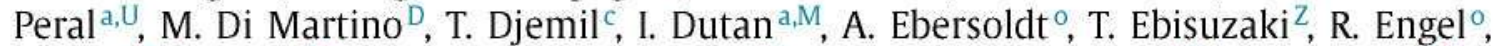
J. Eser ${ }^{\mathrm{b}, \mathrm{D}}$, F. Fenu ${ }^{\mathrm{B}, \mathrm{C}}, \mathrm{S}$. Fernández-González ${ }^{\mathrm{a}, \mathrm{S}}$, J. Fernández-Soriano ${ }^{\mathrm{a}, \mathrm{U}}, \mathrm{S}$. Ferrarese ${ }^{\mathrm{B}, \mathrm{C}}$, M. Flamini ${ }^{\mathrm{E}}$, C. Fornaro ${ }^{\mathrm{E}}$, M. Fouka ${ }^{\mathrm{b}}$, A. Franceschi ${ }^{\mathrm{V}}$, S. Franchini ${ }^{\mathrm{aV}}$, C. Fuglesang ${ }^{\mathrm{aX}}$, T. Fujii ${ }^{\mathrm{K}}$, J. Fujimoto ${ }^{\mathrm{Y}}$, M. Fukushima ${ }^{\mathrm{K}}$, P. Galeotti ${ }^{\mathrm{B}, \mathrm{C}}$, E. García-Ortega ${ }^{\mathrm{a}, \mathrm{S}}, \mathrm{G}$. Garipover, ${ }^{\mathrm{a}, \mathrm{P}}$, E. Gascón ${ }^{\mathrm{a}, \mathrm{S}}$, J. Genci ${ }^{\mathrm{a}, \mathrm{R}}$, G. Giraudo ${ }^{\mathrm{B}}$, C. González Alvarado ${ }^{\mathrm{a}, \mathrm{T}}$, P. Gorodetzky ${ }^{1}$, R. Greg ${ }^{\mathrm{b}, \mathrm{D}}, \mathrm{F}$. Guarino ${ }^{\mathrm{w}, \mathrm{x}}$, A. Guzmán ${ }^{q}, Y$. Hachisu ${ }^{\mathrm{Z}}$, M. Haiduc ${ }^{\mathrm{a}, \mathrm{M}}, \mathrm{B}$. Harlov ${ }^{\mathrm{a}, \mathrm{O}}$, A. Haungs $^{\circ}$, J. Hernández Carretero $^{\mathrm{a}, \mathrm{U}}$, W. Hidber Cruz $^{a, D}$, D. Ikeda ${ }^{K}, N$. Inoue ${ }^{T}, S$. Inoue ${ }^{Z}$, F. Isgrò ${ }^{W, E}, Y$. Itow ${ }^{Q}, T$. Jammer ${ }^{P}$, S. Jeong ${ }^{\mathrm{a}, \mathrm{C}}$, E. Joven $^{\mathrm{a}, \mathrm{W}}$, E.G. Judd ${ }^{\mathrm{b}, \mathrm{B}}$, A. Jung ${ }^{1}$, J. Jochum ${ }^{\mathrm{P}}$, F. Kajino ${ }^{\mathrm{L}}$, T. Kajino ${ }^{\circ}$, S. Kalli ${ }^{\mathrm{f}}$, I. Kaneko ${ }^{\text {, }}$, Y. Karadzhov ${ }^{\mathrm{i}}$, J. Karczmarczyk ${ }^{\mathrm{a}, \mathrm{K}}$, K. Katahira ${ }^{\mathrm{Z}}$, K. Kawai ${ }^{\mathrm{Z}}$, Y. Kawasaki ${ }^{\text {, }}$ A. Kedadra ${ }^{\mathrm{a}}$, H. Khales ${ }^{\mathrm{a}}$, B.A. Khrenov ${ }^{\mathrm{a}, \mathrm{P}}$, Jeong-Sook Kim ${ }^{\mathrm{a}, \mathrm{A}}$, Soon-Wook Kim ${ }^{\mathrm{a}, \mathrm{A}}$, M. Kleifges ${ }^{\circ}$, P.A. Klimov ${ }^{\mathrm{a}, \mathrm{P}}$, D. Kolev ${ }^{\mathrm{i}}$, H. Krantz ${ }^{\mathrm{b}, \mathrm{D}}$, I. Kreykenbohm ${ }^{\mathrm{n}}$, K. Kudela ${ }^{\mathrm{a}, \mathrm{Q}}$, Y. Kurihara ${ }^{Y}$, A. Kusenko ${ }^{\mathrm{X}, \mathrm{b}, \mathrm{F}}$, E. Kuznetsov ${ }^{\mathrm{b}, \mathrm{E}}$, A. La Barbera ${ }^{\mathrm{y}, \mathrm{u}}$, C. Lachaud $^{\mathrm{l}}$, H. Lahmar ${ }^{\mathrm{a}}$, F. Lakhdari ${ }^{g}$, O. Larsson ${ }^{\mathrm{a}, \mathrm{X}}$, J. Lee ${ }^{\mathrm{a}, \mathrm{C}}, \mathrm{J}$. Licandro ${ }^{\mathrm{a}, \mathrm{W}}$, L. López Campano ${ }^{\mathrm{a}, \mathrm{S}}$, M.C. Maccarone ${ }^{\mathrm{y}, \mathrm{u}}$, S. Mackovjak ${ }^{\mathrm{a}, \mathrm{Z}}$, M. Mahdi ${ }^{\mathrm{a}}$, D. Maravilla ${ }^{\mathrm{a}, \mathrm{D}}$, L. Marcelli ${ }^{\mathrm{z}}$, J.L. Marcos ${ }^{\mathrm{a}, \mathrm{S}}$, A. Marini ${ }^{\mathrm{v}}$, W. Marszal ${ }^{\mathrm{a}, \mathrm{K}}, \mathrm{K}$. Martens ${ }^{\mathrm{X}}$, Y. Martín ${ }^{\mathrm{a}, \mathrm{W}}, \mathrm{O}$. Martinez ${ }^{\mathrm{a}, \mathrm{F}}, \mathrm{M}$. Martucci ${ }^{\mathrm{v}}$, G. Masciantonio $^{z}$, K. Mase ${ }^{G}$, M. Mustafa ${ }^{\mathrm{b}, \mathrm{E}}, \mathrm{R}$ Matev $^{\mathrm{i}}$, J.N. Matthews ${ }^{\mathrm{b}, \mathrm{J}}$, N. Mebarki ${ }^{\mathrm{d}}$, G. Medina-Tanco $^{\mathrm{a}, \mathrm{D}}$, M.A. Mendoza ${ }^{\mathrm{a}, \mathrm{G}}$, A. Menshikov ${ }^{\mathrm{o}}$, A. Merino ${ }^{\mathrm{a}, \mathrm{S}}$, J. Meseguer ${ }^{\mathrm{a}, \mathrm{V}}$, S.S. Meyer ${ }^{\mathrm{b}, \mathrm{C}}$, J. Mimouni ${ }^{\mathrm{d}}$, H. Miyamoto ${ }^{B, C}, Y_{\text {. Mizumoto }}{ }^{\circ}$, A. Monaco ${ }^{\mathrm{r}, \mathrm{S}}$, J.A. Morales de los Ríos ${ }^{\mathrm{a}, \mathrm{U}}, \mathrm{S}$. Nagataki ${ }^{\text {, }}$, S. Naitamor ${ }^{\mathrm{b}}$, T. Napolitano ${ }^{\mathrm{v}}$, R. Nava ${ }^{\mathrm{a}, \mathrm{D}}$, A. Neronov ${ }^{\mathrm{a}, \mathrm{Z}}$, K. Nomoto $^{\mathrm{X}}$, T. Nonaka ${ }^{\mathrm{K}}$, T. Ogawa ${ }^{\mathrm{Z}}$,

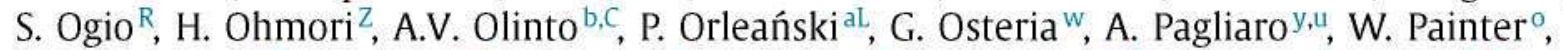
M.I. Panasyuk ${ }^{\mathrm{a}, \mathrm{P}}$, B. Panico ${ }^{\mathrm{w}}$, E. Parizot ${ }^{1}$, I.H. Park ${ }^{\mathrm{a}, \mathrm{C}}$, B. Pastircak $^{\mathrm{a}, \mathrm{Q}}$, , T. Patzak $^{1}$, T. Paul ${ }^{\mathrm{b}, \mathrm{G}}$, I. Pérez-Grande ${ }^{a, V}$, F. Perfetto ${ }^{w, x}$, T. Peter $^{b, A}$, P. Picozza ${ }^{z, A, Z}$, S. Pindado ${ }^{a, V}$, L.W. Piotrowski ${ }^{Z}$,

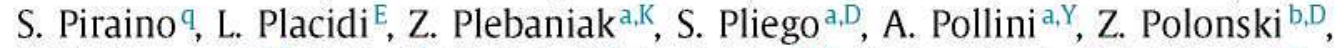
E.M. Popescu ${ }^{\mathrm{a}, \mathrm{M}}$, P. Prat ${ }^{1}$, G. Prévôt ${ }^{1}$, H. Prieto ${ }^{\mathrm{a}, \mathrm{U}}$, G. Puehlhofer ${ }^{\mathrm{q}}$, M. Putis ${ }^{\mathrm{a}, \mathrm{Q}}$, J. Rabanal ${ }^{\mathrm{j}}$, A.A. Radu ${ }^{\mathrm{a}, \mathrm{M}}$, M. Reyes ${ }^{\mathrm{a}, \mathrm{W}}$, M. Rezazadeh ${ }^{\mathrm{b}, \mathrm{C}}$, M. Ricci ${ }^{\mathrm{v}}$, M.D. Rodríguez Frías ${ }^{\mathrm{a}, \mathrm{U}}$, F. Ronga ${ }^{\mathrm{v}}$, G. Roudil $^{\mathrm{m}}$, I. Rusinov ${ }^{\mathrm{i}}$, M. Rybczyński ${ }^{\mathrm{a}, \mathrm{J}}$, M.D. Sabau ${ }^{\mathrm{a}, \mathrm{T}}$, G. Sáez Cano ${ }^{\mathrm{a}, \mathrm{U}}$, H. Sagawa ${ }^{\mathrm{K}}$, 
Z. Sahnoune ${ }^{b}$, A. Saito ${ }^{M}$, N. Sakaki ${ }^{\mathrm{K}}$, H. Salazar ${ }^{\mathrm{a}, \mathrm{F}}$, J.C. Sanchez Balanzara,D, J.L. Sánchez ${ }^{\mathrm{a}, \mathrm{S}}$, A. Santangelo ${ }^{q}$, A. Sanz-Andrés ${ }^{a, V}$, M. Sanz Palomino ${ }^{a, T}$, O. Saprykin ${ }^{a, O}$, F. Sarazin ${ }^{\mathrm{b}, \mathrm{D}}$, M. Sato ${ }^{\mathrm{U}}$, T. Schanz ${ }^{4}$, H. Schieler ${ }^{\circ}$, V. Scotti ${ }^{w, x}$, S. Selmane, D. Semikoz ${ }^{1}$, M. Serra ${ }^{a, w}$,

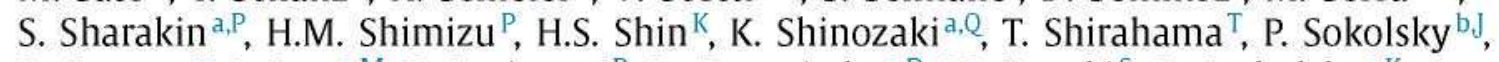
B. Spataro ${ }^{\text {, }}$, I. Stan ${ }^{\mathrm{a}, \mathrm{M}}$, T. Sugiyama ${ }^{\mathrm{P}}$, D. Supanitsky ${ }^{\mathrm{a}, \mathrm{D}}$, M. Suzuki ${ }^{\mathrm{S}}$, B. Szabelska ${ }^{\mathrm{a}, \mathrm{K}}$, J. Szabelski ${ }^{\mathrm{a}, \mathrm{K}}$, N. Tajima ${ }^{\mathrm{Z}}, \mathrm{T}_{\text {. Tajima }}$, Y. Takahashi ${ }^{\mathrm{U}}$, H. Takami $^{\mathrm{Y}}$, M. Takeda ${ }^{\mathrm{K}}$, T. Takizawa $^{\mathrm{Z}}$,

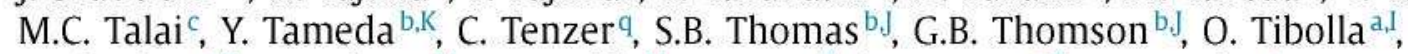

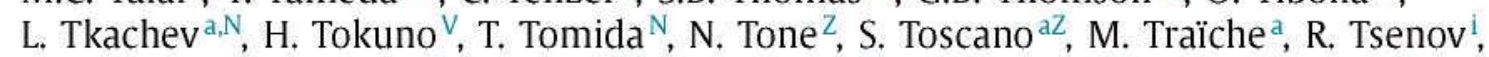
Y. Tsunesada ${ }^{\mathrm{R}}$, K. Tsuno ${ }^{\text {, J. J. Tubbs }}{ }^{\mathrm{b}, \mathrm{E}}, \mathrm{S}$. Turriziani ${ }^{\mathrm{Z}}$, Y. Uchihori ${ }^{\mathrm{H}}, \mathrm{O}$. Vaduvescu ${ }^{\mathrm{a}, \mathrm{W}}$, J.F. Valdés-Galiciaa ${ }^{a, D}$, P. Vallania ${ }^{B, D}$, G. Vankova ${ }^{i}$, C. Vigorito ${ }^{B, C}$, L. Villaseñor $^{\mathrm{a}, \mathrm{E}}$, B. Vlcek ${ }^{\mathrm{a}, \mathrm{U}}$, P. von Ballmoos ${ }^{m}$, M. Vrabel ${ }^{\mathrm{a}, \mathrm{R}}, \mathrm{S}$. Wada ${ }^{\mathrm{Z}}$, J. Watanabe ${ }^{0}$, J. Watts Jr. ${ }^{\mathrm{b}, \mathrm{E}}$, M. Weber $^{\circ}$, R. Weigand Muñoz ${ }^{\mathrm{a}, \mathrm{S}}$, A. Weindl ${ }^{\circ}$, L. Wiencke ${ }^{\mathrm{b}, \mathrm{D}}$, M. Wille ${ }^{\mathrm{n}}$, J. Wilms $^{\mathrm{n}}$, Z. Włodarczyk $^{\mathrm{a} J}$,

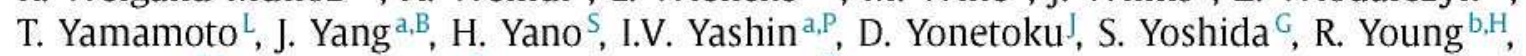
I.S. Zgura ${ }^{\mathrm{a}, \mathrm{M}}$, M.Yu. Zotov ${ }^{\mathrm{a}, \mathrm{P}}$, A. Zuccaro Marchi ${ }^{\mathrm{Z}}$

a Centre for Development of Advanced Technologies (CDTA), Algiers, Algeria

${ }^{b}$ Department of Astronomy, Centre Res. Astronomy, Astrophysics and Geophysics (CRAAG), Algiers, Algeria

'LPR at Department of Physics, Faculty of Sciences, University Badji Mokhtar, Annaba, Algeria

${ }^{d}$ Lab. of Math. and Sub-Atomic Phys. (LPMPS), University of Constantine I, Constantine, Algeria

${ }^{e}$ Laboratory of Theoretical Physics LPT, University of Jijel, Jijel, Algeria

${ }^{\mathrm{f}}$ Department of Physics, Faculty of Sciences, University of M'sila, M'sila, Algeria

${ }^{\mathrm{s}}$ Research Unit on Optics and Photonics, UROP-CDTA, Sétif. Algeria

${ }^{\mathrm{h}}$ Telecom Lab., Faculty of Technology, University Abou Bekr Belkaid, Tlemcen, Algeria

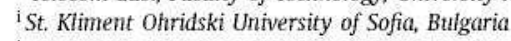

j LAL, Univ Paris-Sud, CNRS/N2P3, Orsay, France

komega, Ecole Polytechnique, CNRS/IN2P3, Palaiseau, France

${ }^{1}$ APC, Univ Paris Diderot, CNRS/IN2P3, CEA/Irfu, Obs de Paris, Sorbonne Paris Cité, France

mIRAP, Université de Toulouse, CNRS, Toulouse, France

${ }^{n}$ ECAP, University of Eriangen-Nuremberg, Germany

${ }^{\circ}$ Karlsruhe Institute of Technology (KIT), Germany

${ }^{\mathrm{P}}$ Experimental Physics Institute, Kepler Center, University of Tubingen, Germany

${ }^{9}$ Institute for Astronomy and Astrophysics, Kepler Center, University of Tubingen, Germany

${ }^{\mathrm{r}}$ Istituto Nazionale di Fisica Nucleare, Sezione di Bari, Italy

'Universita' degli Studi di Bari Aldo Moro and INFN, Sezione di Bari, Italy

'Dipartimento di Fisica e Astronomia, Universita' di Catania, Italy

"Istituto Nazionale di Fisica Nucleare, Sezione di Catania, Italy

"Istituto Nazionale di Fisica Nucleare, Laboratori Nazionali di Frascati, Italy

${ }^{w}$ Istituto Nazionale di Fisica Nucleare, Sezione di Napoli, Italy

x Universita' di Napoli Federico II, Dipartimento di Scienze Fisiche, Italy

y INAF - Istituto di Astrofisica Spaziale e Fisica Cosmica di Palermo, Italy

${ }^{2}$ Istituto Nazionale di Fisica Nucleare, Sezione di Roma Tor Vergata, Italy

A Universita' di Roma Tor Vergata, Dipartimento di Fisica, Roma, Italy

${ }^{\mathrm{B}}$ Istituto Nazionale di Fisica Nucleare, Sezione di Torino, Italy

'Dipartimento di Fisica, Universita' di Torino, Italy

D Osservatorio Astrofisico di Torino, Istituto Nazionale di Astrofisica, Italy

${ }^{E}$ UTIU, Dipartimento di Ingegneria, Rome, Italy

F DIETI, Universita' degli Studi di Napoli Federico II, Napoli, Italy

${ }^{\mathrm{G}}$ Chiba University, Chiba, Japan

${ }^{\mathrm{H}}$ National Institute of Radiological Sciences, Chiba, Japan

${ }^{1}$ Kinki University, Higashi-Osaka, Japan

${ }^{\mathrm{J}}$ Kanazawa University, Kanazawa, Japan

${ }^{\mathrm{K}}$ Institute for Cosmic Ray Research, University of Tokyo, Kashiwa, Japan

${ }^{\mathrm{L}}$ Konan University, Kobe, Japan

${ }^{M}$ Kyoto University, Kyoto, Japan

N Shinshu University, Nagano, Japan

${ }^{-}$National Astronomical Observatory, Mitaka, Japan

${ }^{\mathrm{P}}$ Nagoya University, Nagoya, Japan

eInstitute for Space-Earth Environmental Research, Nagoya University, Nagoya, Japan

${ }^{\mathrm{R}}$ Graduate School of Science, Osaka City University, Japan

SInstitute of Space and Astronautical Science/JAXA, Sagamihara, Japan

TSaitama University, Saitama, Japan

${ }^{\mathrm{H}}$ Hokkaido University, Sapporo, Japan

${ }^{\mathrm{V}}$ Interactive Research Center of Science, Tokyo Institute of Technology, Tokyo, Japan

wihon University Chiyoda, Tokyo, Japan

$\mathrm{x}$ University of Tokyo, Tokyo, Japan

${ }^{Y}$ High Energy Accelerator Research Organization (KEK), Tsukuba, Japan

${ }^{2}$ RIKEN, Wako, Japan

aA Korea Astronomy and Space Science Institute (KASI), Daejeon, Republic of Korea

${ }^{\mathrm{aB}}$ Ewha Womans University, Seoul, Republic of Korea

${ }^{a}$ Sungkyunkwan University, Seoul, Republic of Korea

aD Universidad Nacional Autónoma de México (UNAM), Mexico

${ }^{a}$ Universidad Michoacana de San Nicolas de Hidalgo (UMSNH), Morelia, Mexico

${ }^{a f}$ Benemérita Universidad Autónoma de Puebla (BUAP), Mexico 
${ }^{a G}$ Centro de Desarrollo Aeroespacial, Instituto Politécnico National (CDA-IPN), Mexico

${ }^{\mathrm{aH}}$ Universidad Autónoma de Chiapas (UNACH), Chiapas,Mexico

al Centro Mesoamericano de Fisica Teórica (MCTP), Mexico

a) Institute of Physics, Jan Kochanowski University, Kielce, Poland

${ }^{\mathrm{aK}}$ National Centre for Nuclear Research, Lodz, Poland

al Space Research Centre of the Polish Academy of Sciences (CBK), Warsaw, Poland

aM Institute of Space Science ISS, Magurele, Romania

aN Joint Institute for Nuclear Research, Dubna, Russia

${ }^{\text {aO }}$ Central Research Institute of Machine Building. TsNIMash, Korolev, Russia

${ }^{\mathrm{P}}$ Skobeltsyn Institute of Nuclear Physics, Lomonosov Moscow State University, Russia

al Institute of Experimental Physics, Kosice, Slovakia

aR Technical University Kosice (TUKE), Kosice, Slovakio

as Universidad de León (ULE), León, Spain

aT Instituto Nacional de Técnica Aeroespacial (INTA), Madrid, Spain

${ }^{a U}$ Universidad de Alcalá (UAH), Madrid, Spain

av Universidad Politécnia de Madrid (UPM), Madrid, Spain

${ }^{a W}$ Instituto de Astrofisica de Canarias (IAC), Tenerife, Spain

${ }^{a}$ KTH Royal Institute of Technology, Stockholm, Sweden

aY Swiss Center for Electronics and Microtechnology (CSEM), Neuchâtel, Switzerland

${ }^{a}$ ISDC Data Centre for Astrophysics, Versoix, Switzerland

bA Institute for Atmospheric and Climate Science, ETH Zürich, Switzerland

${ }^{\mathrm{bB}}$ Space Science Laboratory, University of California, Berkeley, USA

${ }^{\mathrm{bC}}$ University of Chicago, USA

bD Colorado School of Mines, Golden, USA

bE University of Alabama in Huntsville, Huntsville, USA

bF NASA Jet Propulsion Laboratory, Pasadena, USA

${ }^{b G}$ Lehman College, City University of New York (CUNY), USA

${ }^{\mathrm{bH}}$ NASA - Marshall Space Flight Center, USA

bl Vanderbilt University, Nashville, USA

b University of Utah, Salt Lake City, USA

${ }_{\mathrm{bK}}$ Department of Engineering Science, Faculty of Engineering, Osaka Electro-Communication University, Neyagawa-shi, Osaka, Japan

\begin{abstract}
A B S T R A C T
EUSO-TA is a ground-based telescope, installed at the Telescope Array (TA) site in Black Rock Mesa, Utah, USA. This is the first detector to successfully use a Fresnel lens based optical system and multi-anode photomultipliers ( 64 channels per tube, 2304 channels encompassing a $10.6^{\circ} \times 10.6^{\circ}$ field of view) for detection of Ultra High Energy Cosmic Rays (UHECR). The telescope is located in front of one of the fluorescence detectors of the TA experiment. Since its installation in 2013, the detector has observed several ultra-high energy cosmic ray events and, in addition, meteors. The limiting magnitude of 5.5 on summed frames ( $\sim 3 \mathrm{~ms}$ ) has been established. Measurements of the UV night sky emission in different conditions and moon phases and positions have been completed. The performed observations serve as a proof of concept for the future application of this detector technology.
\end{abstract}

\section{Introduction}

JEM-EUSO is a proposed space-borne mission concept for the detection of cosmic rays of the highest energies [1]. It is designed to observe the ultraviolet (UV) fluorescence light from Extended Air Showers (EAS) generated by cosmic rays in the atmosphere with a Fresnel lens based optical system and a super-fast single photon counting camera. Compared to existing ground-based experiments, JEM-EUSO would be able to observe a much larger volume of the atmosphere, significantly increasing the number of detected events at the highest energies and thus the available data for the localisation of their sources in the Universe.

The first attempt to use Fresnel lenses to observe EAS was made in late sixties near Ithaca, USA, resulting in measurements of a Xenon flasher calibration light source [2]. The first observations in the framework of the EUSO concept have been performed with the ground-based experiment EUSO-TA, which is the main focus of this paper. The flight of EUSO-BALLOON, with similar design, followed in 2014 [3] and successfully observed laser-simulated EAS, LED flashes and the night-time UV emission of Earth. In 2017 a super pressure balloon with upgraded electronics - EUSO-SPB - has flown with a pioneering aim of observing UV light from EAS looking down on the atmosphere. Currently, another mission is in the final stage of preparation - Mini-EUSO - designed to be hosted inside the International Space Station, observing the atmosphere through a UV-transparent window [4]. Mini-EUSO will be sensitive to EAS with primary energies above $10^{21} \mathrm{eV}$, meteors, strange quark matter and atmospheric events such as Transient Luminous Events (TLEs). It will also produce a detailed UV map of the nighttime Earth. It will be followed by K-EUSO - a mission led by Russian Space Agency, placing an UHECR observatory on board the Russian Segment of the ISS [5,6].

EUSO-TA is a ground-based telescope located at Black Rock Mesa, Utah, USA at the site of one of the fluorescence detectors of the Telescope Array (TA) experiment [7] (Fig, 1). From there it observes, simultaneously with $\mathrm{TA}$, both artificial calibration light and cosmic ray events, allowing for tests of the EUSO technology, calibration of the detector and reduction of the systematic uncertainties of the measurements. The location and pointing direction allows for observation of TA's Central Laser Facility (CLF) and Electron Light Source (ELS) (Fig. 2).

\section{EUSO-TA instrument}

The $1 \mathrm{~m}^{2}$ EUSO-TA Fresnel lenses $\left(0.92 \mathrm{~m}^{2}\right.$ active area) are fabricated from UV transmitting polymethyl-methacrylate (PMMA). The baseline design of the optics is shown in fig. 3 with ray tracing for 


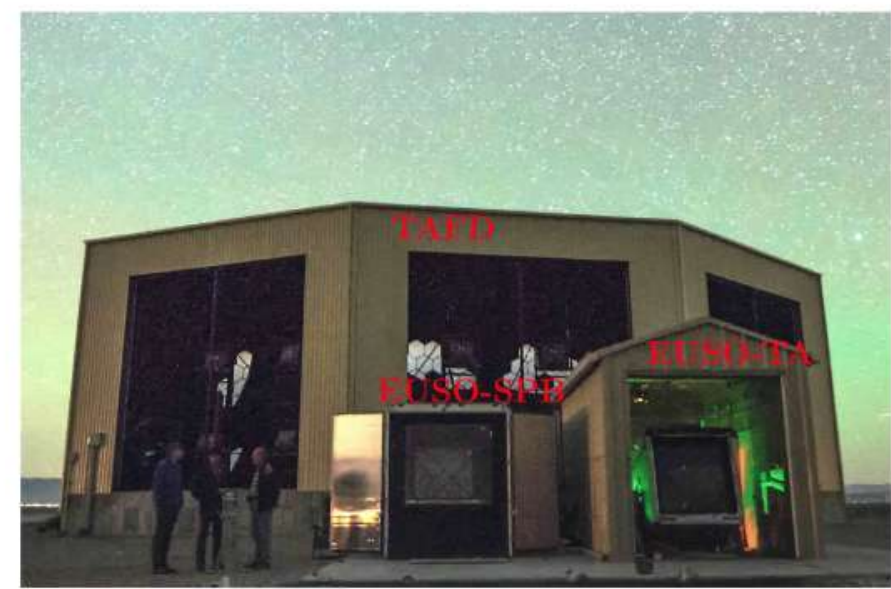

Fig. 1. EUSO-TA (front right), EUSO-SPB (front middle, temporarily installed on-site before its flight) and Telescope Array Fluorescence Detector (TAFD, back) (photography by M. Mustafa).

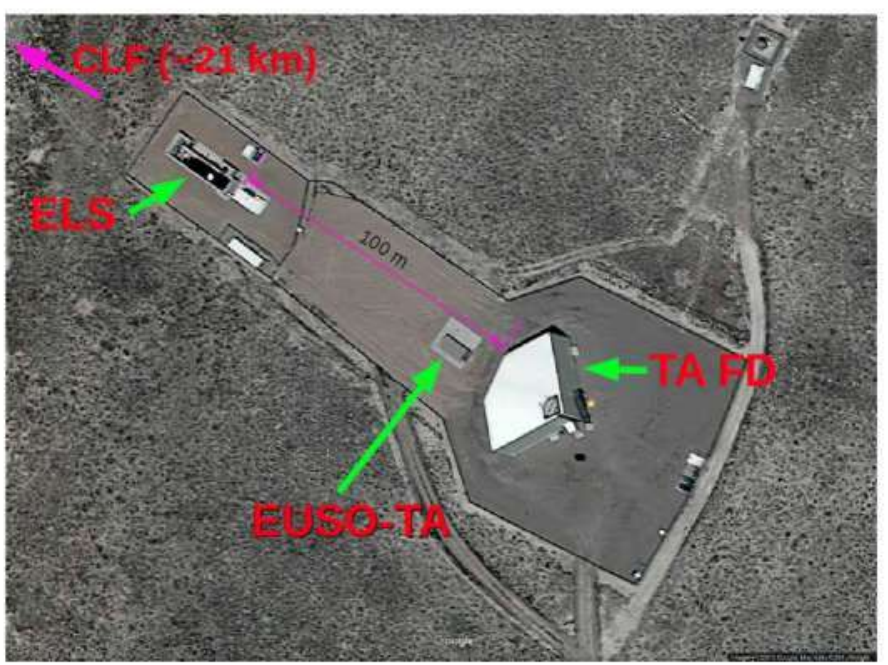

Fig. 2. The position of EUSO-TA on the Telescope Array Fluorescence Detector site in Black Rock Mesa, Utah, USA. The EUSO-TA container is placed directly in front of the TAFD. In its line of sight are the Electron Light Source and the Central Laser Facility instruments of TA. Original photography taken from Google Maps.

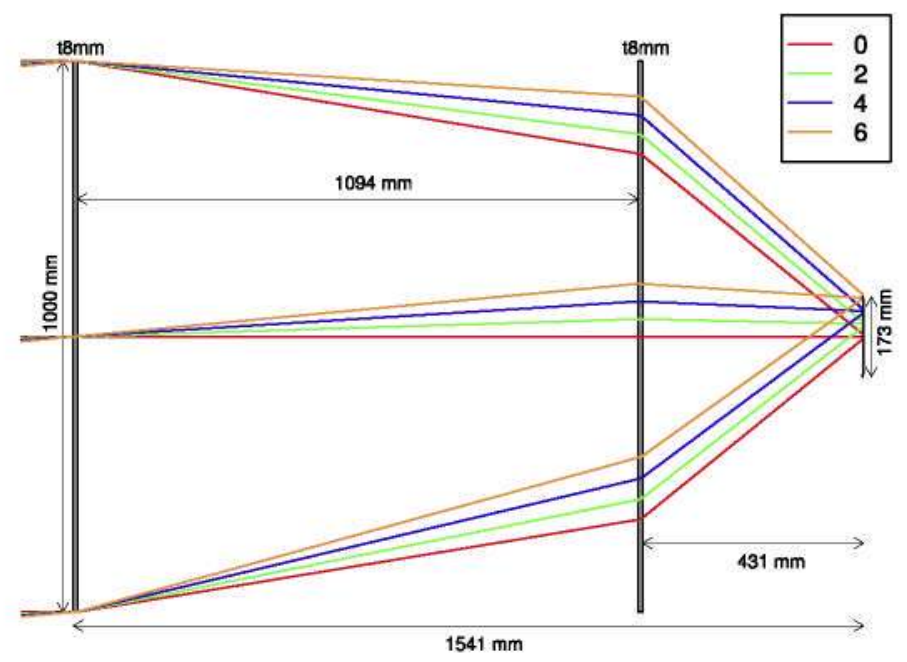

Fig. 3. The design of the EUSO-TA optics. The ray tracing is shown for different incident angles of $0^{\circ}, 2^{\circ}, 4^{\circ}$ and $6^{\circ}$, respectively. incident angles of $0^{\circ}, 2^{\circ}, 4^{\circ}$ and $6^{\circ}$ with respect to the optical axis, covering a $10.6^{\circ}$ (in elevation) $\times 10.6^{\circ}$ (in azimuth) field of view.

The lenses (Fig. 4, left) focus light onto the $17.3 \mathrm{~cm} \times 17.3 \mathrm{~cm}$ Photo Detector Module (PDM), composed of 36 Multi-Anode Photomultiplier Tubes (MAPMTs) [8] each containing 64 anodes, for a total of 2304 pixels (Fig. 4, right), covered by BG3 filters. Four MAPMTs form an EC-Unit, each with a dedicated Cockroft-Walton based High Voltage Power Supply. The operating voltage of the MAPMTs is $1000 \mathrm{~V}$, and is automatically reduced on scale of microseconds in the case of high incident photon flux.

Each MAPMT is read out by one of the SPACIROC1 ASICs, which are distributed over 6 EC-ASIC boards [9]. The single pulse resolution of the ASICs is $\sim 30$ ns. This implies a saturation at about 28 counts (photoelectrons) for each frame - one Gate Time Unit (GTU) - of $2.3 \mu \mathrm{s}$. The frame is preceded by $200 \mathrm{~ns}$ of dead time. This dead time has been reduced to $50 \mathrm{~ns}$ in the next generation ASIC - SPACIROC3 - which has a single pulse resolution of $\sim 5$ ns and thus, being able to count more photons in given period of time, a higher signal to noise ratio. SPACIROC3 has already been used in both EUSO-SPB and Mini-EUSO. EUSO-TA will be upgraded to SPACIROC3 during 2018.

The digitised counts from 6 EC-ASIC boards are read into a ring buffer on the PDM board. This buffer can accommodate 128 GTUs and is read out following an external or internal trigger. One of the main tasks of the PDM board is to perform high-speed, firstlevel triggering (L1) and then forward the selected packet of 128 frames to the Cluster Control Board (CCB). EUSO-TA is usually operated with an external trigger from TAFD. Although implemented in EUSO-TA, the L1 trigger is not optimised for the ground-based configuration due to geometrical constraints (see Section 4.4).

The CCB, which contains a second-level trigger board [10], CPU, Clock board (CLKB), GPS, house keeping and low voltage power supply [11] forms the Data Processor (DP) unit [12]. The exchange of information is made with encapsulated packets. The amount of information contained in a packet increases with the level of processing, the final packet stored by the $\mathrm{CPU}$ on a hard disk contains counts from ASICs, additional information from the PDM board, CCB, CLKB and GPS data [13]. The whole data readout scheme is summarised in Fig. 5.

The focal surface and lenses are mounted on a stand with an adjustable elevation angle between $0^{\circ}$ and $30^{\circ}$. The stand is installed in a container placed in front of the Telescope Array Fluorescence Detector (TAFD) telescope, but below its field of view. Apart from protecting the detector and additional electronics from weather conditions, the walls of the container reduce the stray light entering the instrument.

\section{EUSO-TA observation campaigns}

The EUSO-TA lenses and mechanical structure were installed in TAFD site in Black Rock Mesa, Utah, USA in March 2013. After the installation, initial tests of the optics and mechanics were performed using a single MAPMT with a temporary readout scheme.

Prior to the commissioning of the complete EUSO-TA instrument, its lenses and mechanics were used in the one-pixel UHECR detector "FAST" tests in April and June 2014 [14]. The EUSO-TA PDM and readout system were installed in February-March 2015. Since then, 5 observational campaigns with EUSO-TA have been performed, 4 in 2015 and 1 in 2016. More than 136 hours of observation have been acquired using the TAFD external trigger, thus allowing for coincident detection of UHECR events in the overlapping field of view of the two instruments.

More than 21 hours of data were dedicated to observations using different triggers, usually for synchronising with laser shots, LED flashes and tests of the internal L1 trigger of EUSO experiments. The L1 trigger is tuned to future in-orbit EUSO observa- 

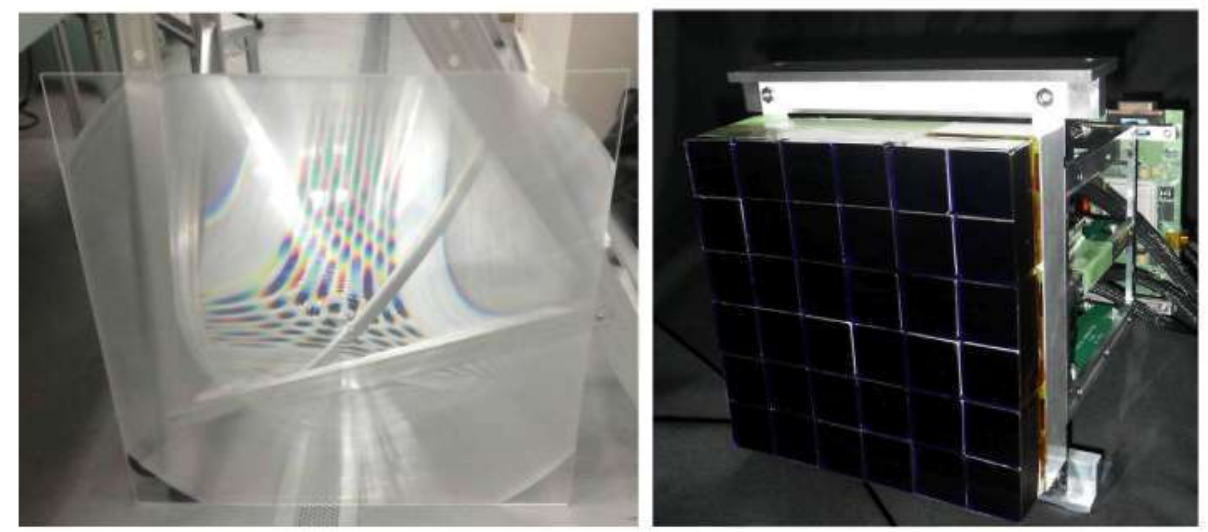

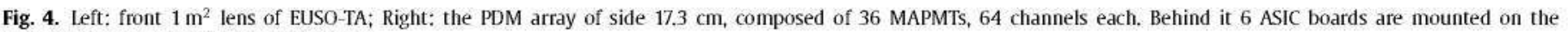
PDM frame connected to the PDM board.

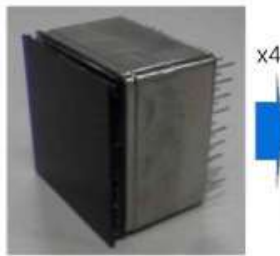

MAPMT module

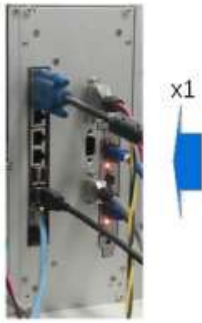

CPU

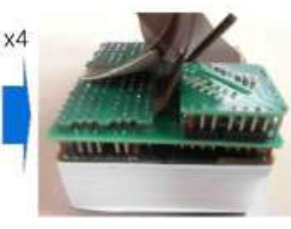

EC-unit (4 MAPMTs)

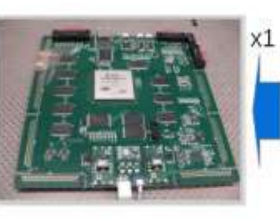

CCB

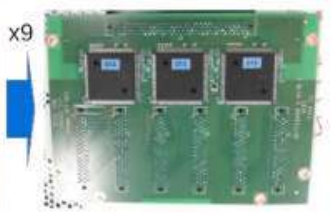

EC-ASIC board $\times 6$

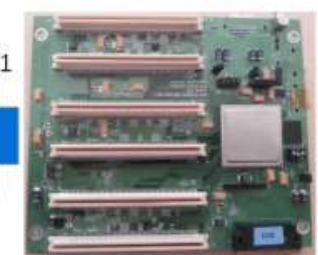

PDM board
Fig. 5. Scheme of the signal flow in the EUSO-TA experiment. The labels over arrows denote signal from how many subsystems of the previous level is included in the subsystem of the next level.

tions, for which the timescale of an EAS crossing the field of view is much longer, and thus it is not optimal for ground-based observations with the same time resolution. For this reason, the external trigger from TAFD was also implemented in EUSO-TA. However, the controlled signals produced by the laser allowed us to analyse the efficiency of the L1 trigger and fine-tune it for the application in EUSO-SPB and Mini-EUSO.

In October 2016, the EUSO-SPB telescope was transported to the EUSO-TA site with a specially modified trailer for ground-based tests and calibration. This allowed for simultaneous observations with the two detectors sharing the same design, the main difference being the upgraded SPACIROC3 ASICS in EUSO-SPB.

\section{Results}

\subsection{Sky background}

The average sky emission ${ }^{1}$ on the EUSO-TA focal surface for a typical dark, cloudless night period (16 $6^{\text {th }}$ of May, 2015, from 9:13 to $9: 29$ UTC) was 1.43 counts per $2.3 \mu$ s (Fig. 6, left). The back-

\footnotetext{
${ }^{1}$ Here "sky emission" is understood to be light registered by the detector excluding distinguishable light sources.
}

ground histogram consists of measurements of pixels with varying efficiencies, and hence varying count distributions, In this way, the overall distribution deviates from Poissonian for high values. The number of counts on a single pixel, which is sensitive to single photons, is well described by the Poisson distribution, as can be seen on Fig. 6, right. This sample pixel has an average number of counts per frame on 100,000 frames equal to 1,28 . In the simplest case, the counts in this pixel would have to exceed $3 \sigma$ threshold of about 5.8 counts to detect a signal. However, the phenomena of observational interest extend over many pixels, lowering this requirement when using more sophisticated detection algorithms.

The baseline background depends on a number of factors such as the time of the night, moon phase, time of the year and the presence of clouds and aerosols. In Fig. 7 the variation of the average number of counts on the whole focal surface is shown during one slightly cloudy night (16 $6^{\text {th }}$ of May, 2015), with a baseline background level higher than 2 counts. The smooth variation is interrupted by clouds passing through the field of view, as shown in Fig. 8. In the case of observations of the sky from the ground, clouds can both decrease and increase the background. The decrease can come from the eclipsing of the nightglow, zodiacal light, milky way and single stars. The increase can come from the reflection of the artificial ground-based lights or from the scattering of the moonlight in moonlit nights. In the case shown, clouds decrease the number of counts, as they block radiation from the nightglow and astronomical objects.

\subsubsection{Flat fielding}

It is a common practice in scientific imaging to equalise pixel sensitivities during data processing. This is especially important for a photomultiplier based photon counter, for which pixels can vary significantly in their efficiencies, For this purpose, flat fielding is often employed. This involves illuminating the telescope with a uniform, Lambertian light source. To achieve this for EUSO-TA, a large reflective flat screen illuminated by a diffused LED was used. The $2.44 \mathrm{~m} \times 2.44 \mathrm{~m}$ flat screen was covered with Tyvek and placed $6.5 \mathrm{~m}$ in front of the EUSO-TA front lens. The LED was placed in the centre of the front lens, emitting light of wavelength $375 \mathrm{~nm}$ through a $3.2^{\circ}$ neutral density filter. The non-uniformity of the detector illumination coming from this setup is small compared to the uncertainties from other sources, mainly due to the fact that the detector was focused at infinity [15].

The flat fielding drastically reduces the width of the pixel counts distribution. On a sample averaged sky frame generated from 12,800 GTUs, $^{2}$ the width of the distribution before flat field-

\footnotetext{
${ }^{2}$ Usually we are using multiples of 128 for frame stacking, since one full packet of data continuous in time consists of 128 frames.
} 

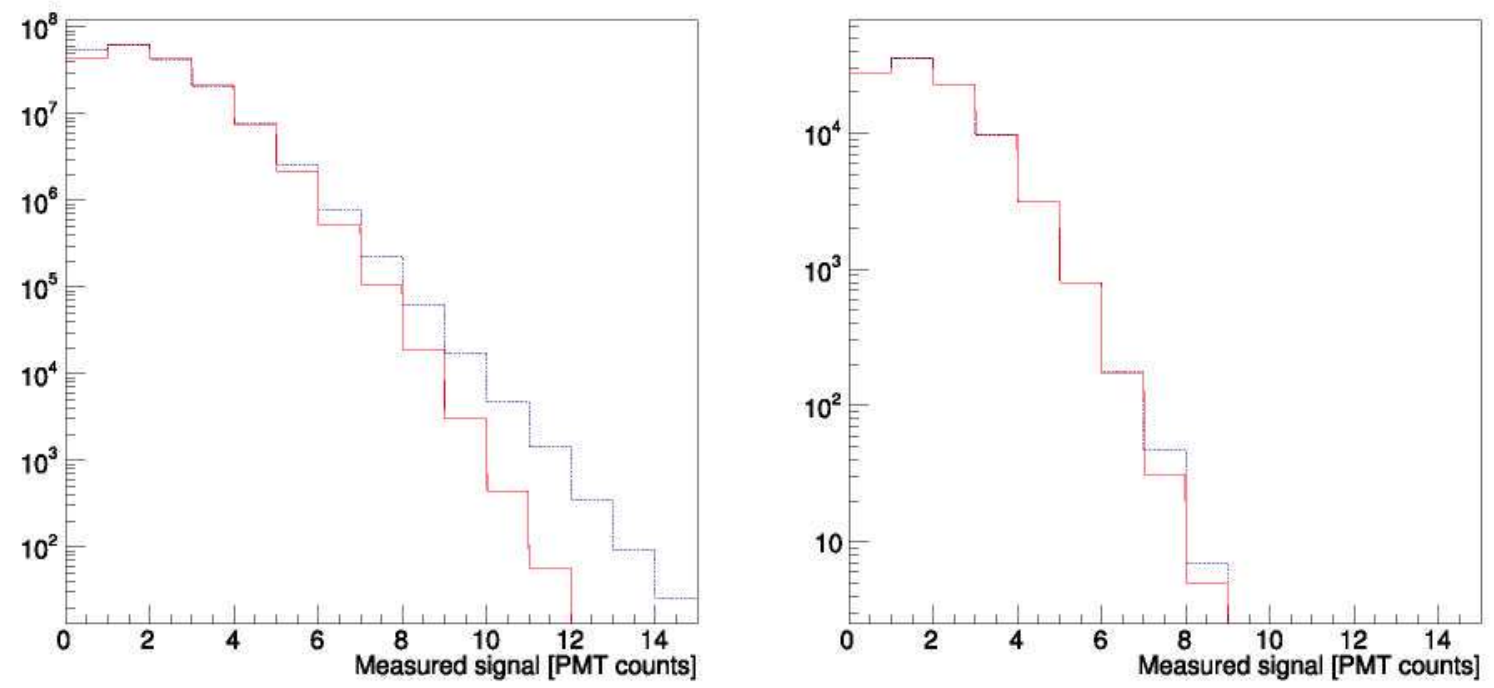

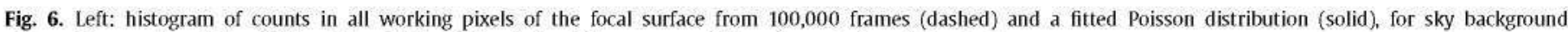
observations on the night of 16th of May, 2015. Right: similar histogram consisting of counts in a single pixel.

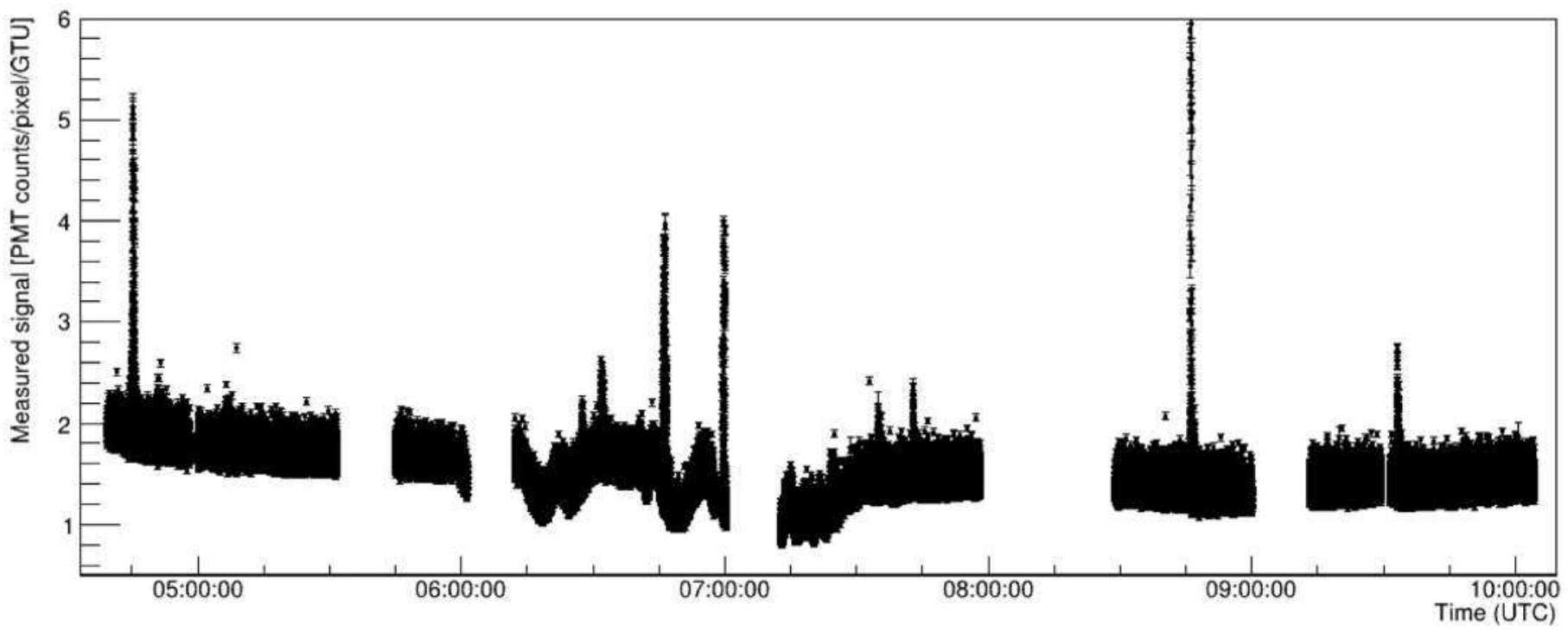

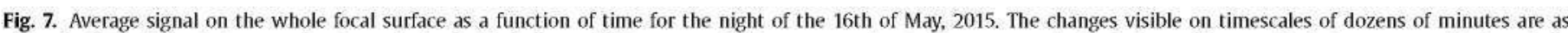

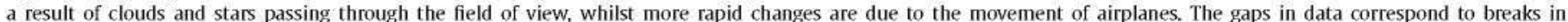
observations. The X-axis spans roughly from 22:00 to 4:00 of local "Mountain Time" (UTC-06:00).

ing was $24.6 \%$, while after it dropped to $9.3 \%$ (Fig. 9). It is important to note that the photographed sky is not completely uniform (due to stars and diffuse sky light sources), so quoted numbers are just a relative estimation of the improvement.

\subsection{Star observations}

Stars can be used as point sources to analyse the sensitivity, the extent of the field of view and the point spread function (PSF) of the detector. EUSO-TA can observe stars up to $M_{B} \simeq 5.5$ (catalogue magnitude in Johnson B filter [16]) on sums of 1280 frames (about $3.2 \mathrm{~ms}$ observation time). This limiting magnitude is a very approximate measure of EUSO-TA sensitivity, since it depends largely on the spectral properties of the star in question and atmospheric conditions at the time of observation (the EUSO-TA spectral window is far from standard Johnson filters). Moreover, the small number of sufficiently bright stars in the field of view do not allow for a precise estimate.
Frame stacking can be used to achieve a better signal to noise ratio, thanks to negligible movement of the stars on the sky for short observation timescales compared to the angular size of a pixel. An example of 1280 stacked frames with a few stars clearly visible is shown in Fig. 10. The brightest star positions are marked on the image following the Hipparcos catalogue [17].

The very wide field of view results in an asymmetric PSF in regions of the focal surface far from the optical axis due to influence of aberrations such as coma or astigmatism. In this way, a complete analysis would be based on fitting the parameters of a PSF model derived from theory and ray-tracing simulations to the images of the stars in different positions on the frame, which is a demanding and difficult task even in detectors with much finer angular resolution [18]. An approach making use of an elliptical Gaussian profile fit gives an asymmetric PSF (Fig. 11) with an average FWHM of $2.98 \pm 0.07$ and $2.46 \pm 0.04$ pixels for the major and minor axes of the ellipse, respectively [19]. This result is well within the requirements for observations of UHECR showers. However, the near-constant asymmetry of the ellipse regardless of the position 

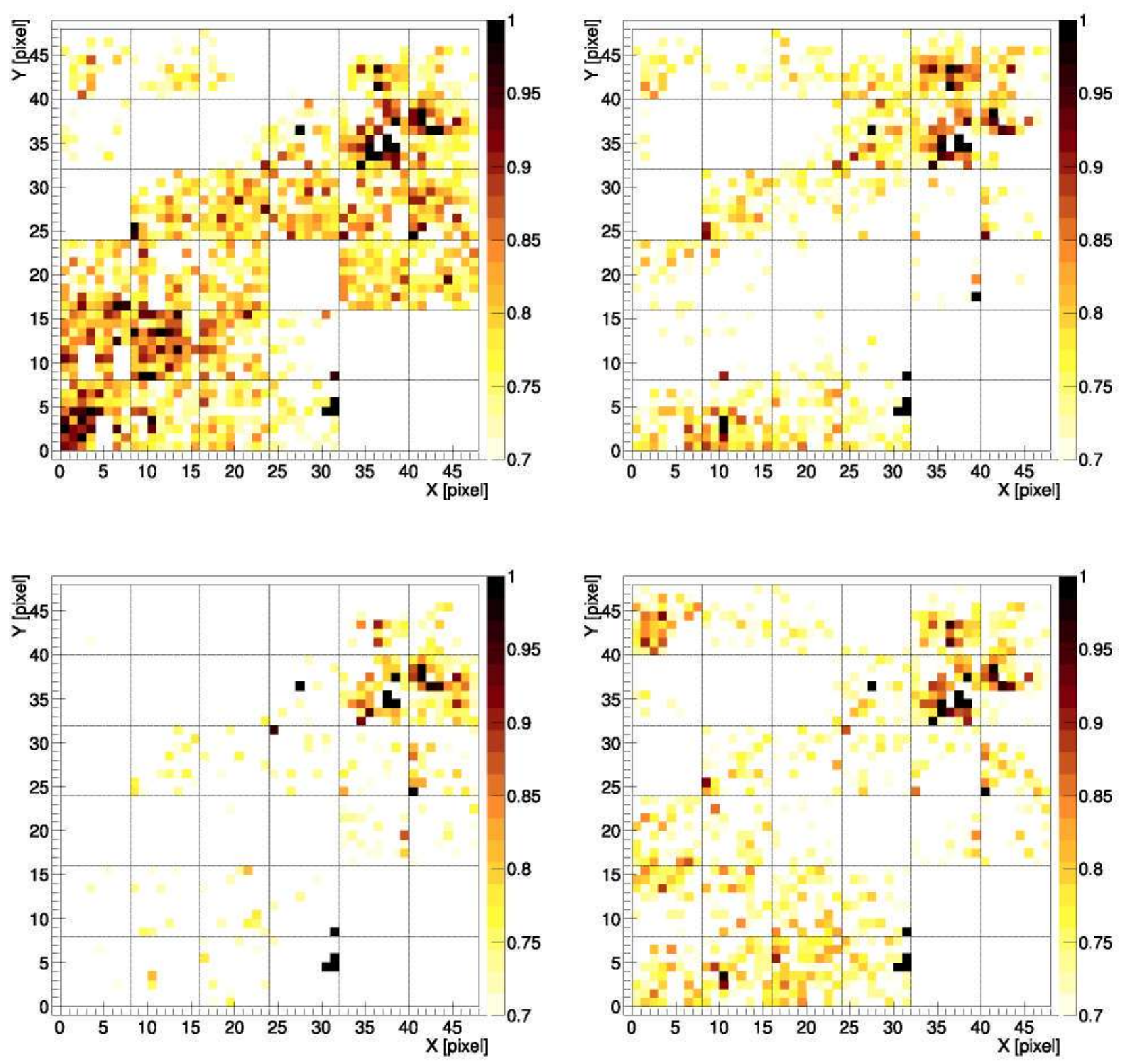

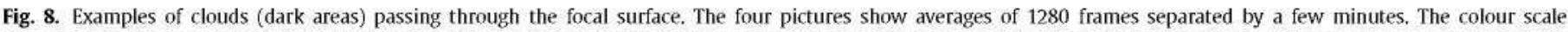

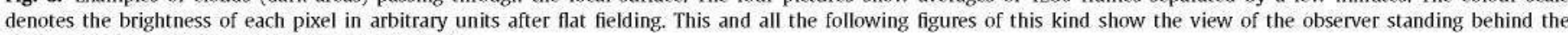
instrument and looking at the same part of the sky.

of the star image on the focal surface suggests a misalignment of the planes of the focal surface and the lenses. This effect likely hides the aforementioned aberrations of the PSF.

The small PSF observed for stationary sources such as stars, but also for laser signals, is important for future, space-based observations of UHECR. Current ground-based experiments employ optics with much larger pixel and PSF size, which would result in poor performance for EAS observations at distances of hundreds of kilometres. Auger's fluorescence detectors have hexagonal pixels with side to side distance of $45.6 \mathrm{~mm}$, corresponding to $1.5^{\circ}$ field of view [20]. Similarly, TAFD pixels are hexagonal in shape with a distance of $60 \mathrm{~mm}$ between the parallel side and cover $1.1^{\circ} \times 1.0^{\circ}$ patch of the sky [21]. For comparison, with EUSO-TA a distant source image, such as that from a star, fits within a rectangle of $3 \times 3$ pixels, each a square of $0.288 \mathrm{~cm}$ side, and angular size of $\sim 0.19^{\circ}$.

The observation of stars allows for astrometry, i.e. determination of the detector's pointing direction in celestial coordinates. For
EUSO-TA this was also used to compute the field of view, resulting in $10.6^{\circ} \pm 0.3^{\circ}$ in both dimensions. Again, the uncertainty is mostly due to the small number of bright stars within the range of the detector, which in turn makes a more sophisticated estimation of the parameters, such as those of a polynomial description of the optical distortion, difficult.

\subsection{Slow phenomena}

Whilst EUSO-TA is designed for observations of microsecondscale events, it can also monitor phenomena taking place on much longer timescales. The most common are flashes from airplanes and sunlight reflected by satellites. Satellite observations can be used as a proof of concept for the plans of space debris remediation with future, orbital EUSO-like experiments [22].

EUSO-TA has also observed meteors, as can be seen in Fig. 12. The magnitudes of three of the observed meteors are shown in Table 1. Such observations are unique due to their microseconds 


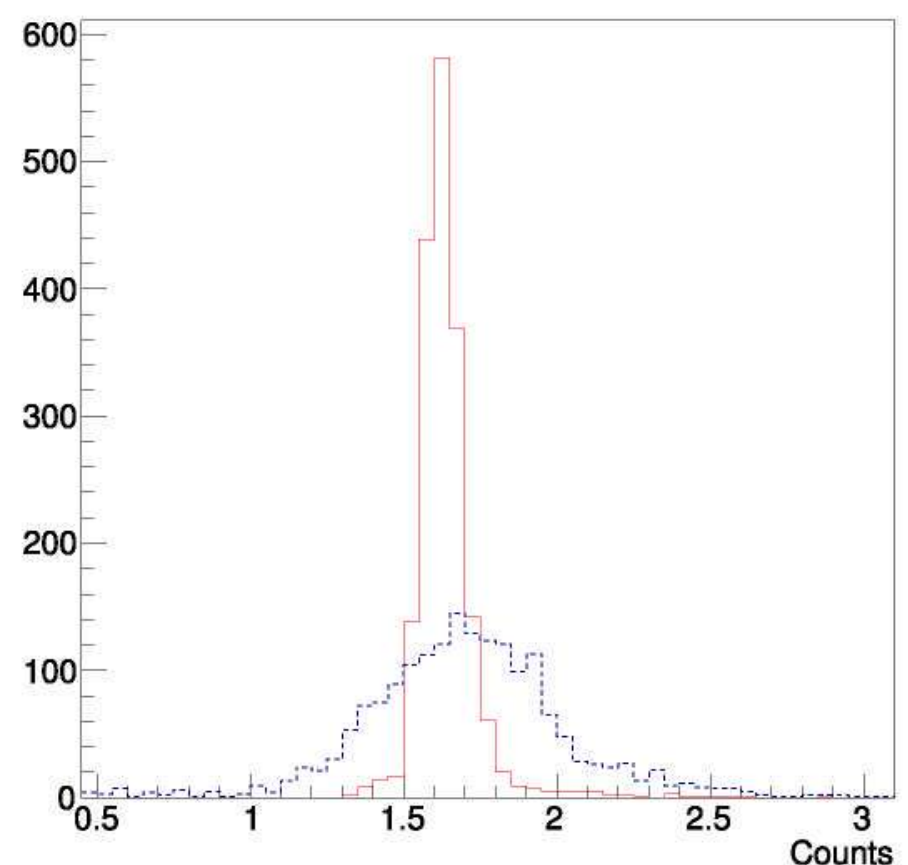

Fig. 9. Histogram of sky background pixel counts on an average of 12,800 frames before (dashed) and after (solid) flat fielding.

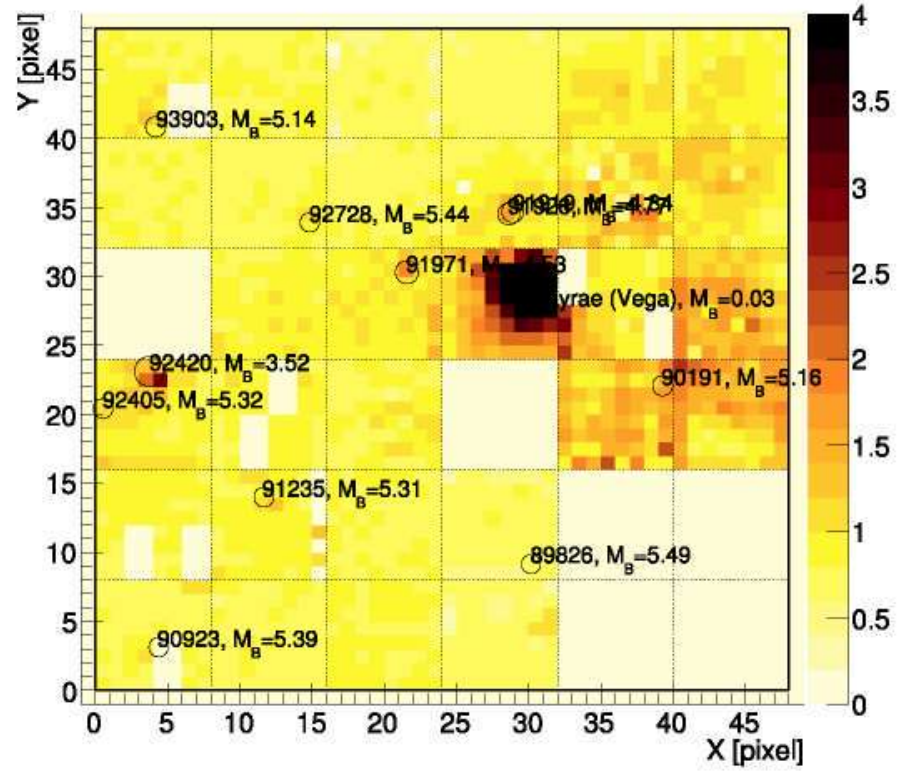

Fig. 10. Sum of 1280 frames acquired with EUSO-TA with the position of the brightest stars superimposed, using the Hipparcos catalogue. Each star is labelled with the catalogue number or the star name in case of Vega, followed by the star's magnitude in the Johnson B filter for objects of $M_{B}<5.5$. The colour scale denotes the brightness of each pixel in arbitrary units after flat fielding. Zero values (bright color) correspond to non-functioning pixels.

Table 1

Apparent magnitude of three meteors as observed by EUSO-TA

\begin{tabular}{lll}
\hline Date & Lowest magnitude & Highest magnitude \\
\hline 2015.09 .18 & $1.59 \pm 0.20$ & $5.27 \pm 0.20$ \\
2015.11 .12 & $2.40 \pm 0.11$ & $4.06 \pm 0.11$ \\
2015.11 .13 & $1.91 \pm 0.03$ & $3.04 \pm 0.05$ \\
\hline
\end{tabular}

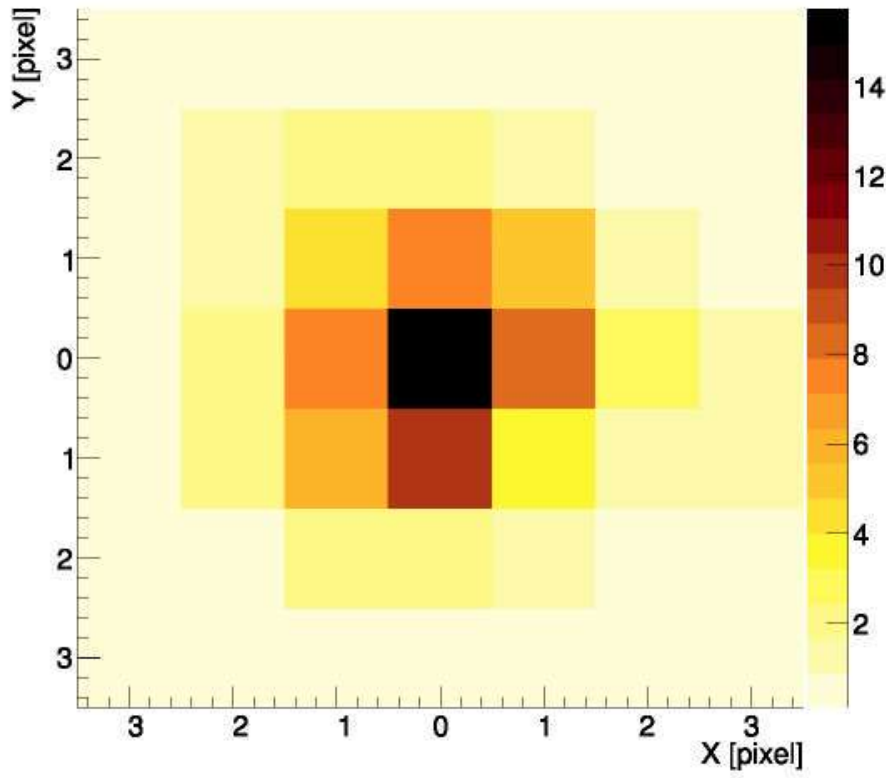

Fig. 11. Sum of 12,800 frames (about $1 \mathrm{~min}$ ) of observations of a typical star for the purpose of PSF estimation. Colours denote the percentage of a signal in the specific bin. This star has Hipparcos catalogue number 100,453 with brightness $\mathrm{M}_{\mathrm{B}}=2.9$.

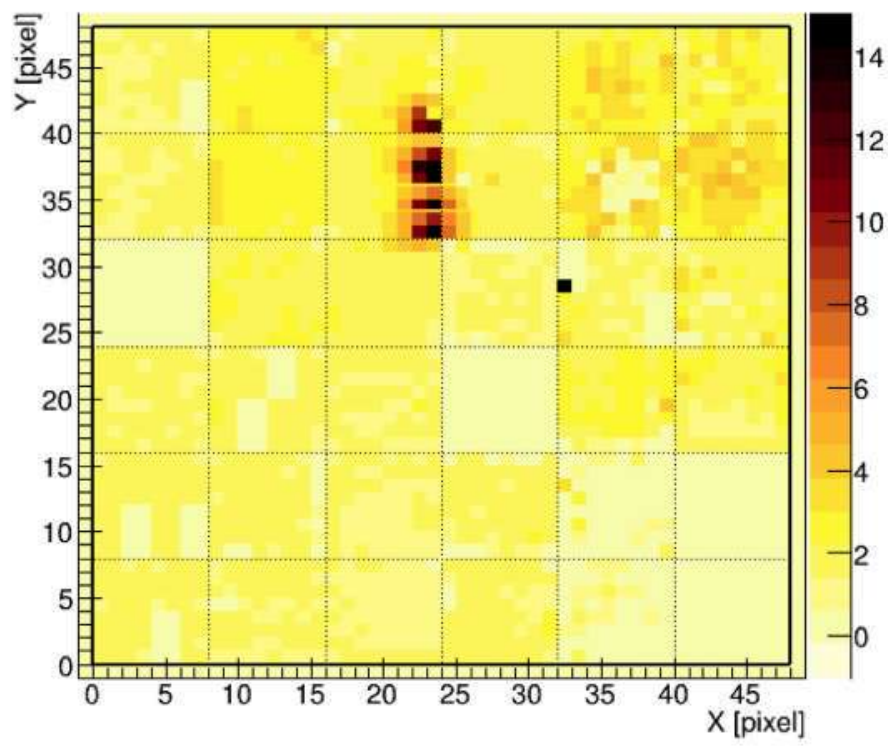

Fig. 12. A meteor track detected by EUSO-TA on the night 12th November, 2015 The picture shows the overlap of four averages of 1280 frames $(0.9 \mathrm{~s}$ elapsed from the start to the end of the observation, $12.8 \mathrm{~ms}$ total integration time). The color scale denotes the uncalibrated detector counts. The apparent magnitude of the meteor in collected data varied from $M_{B}=2.4$ to $M_{B}=4.06$.

to seconds time resolution. This property can be useful to improve our capability to detect the details of the ablation processes undergone by the meteoroid during its passage through the atmosphere. In other words, EUSO-TA can produce meteor lightcurves of much better time resolution than other detectors usually doing meteor observations. In addition, this is related to a better estimation of the meteor speed and more precise determination of the original heliocentric orbit of the meteoroid if we were to perform multidetector observations in the future.

High time resolution and large field of view is important also for the possible detection of a phenomenon similar in appearance to meteors - strangelets [23]. These nuggets of strange matter are predicted to interact with the atmosphere producing light, however with a different lightcurve than that of meteors due to the 


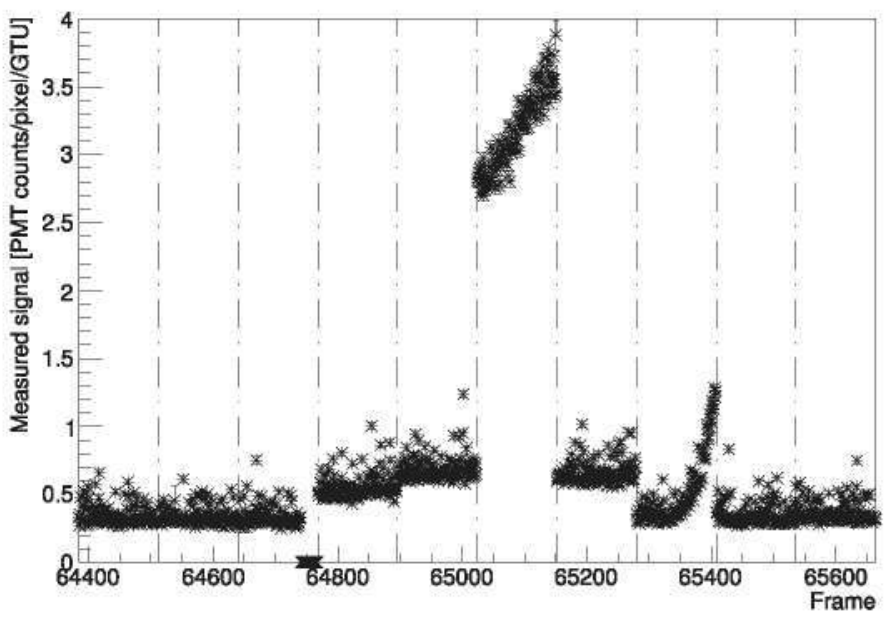

Fig. 13. The lightcurve of the average number of counts per $2.3 \mu$ s on the whole PDM during a thunderstorm passing close by. The lightcurve is not continuous. Vertical lines show the transition between packets of data. Each packet is a continuous acquisition encompassing $320 \mu \mathrm{s}$, however the time between packets depends on incoming triggers and in this case was of order of dozens of milliseconds.

fact that they do not fragment or lose mass, After 90 cumulative days of observations, EUSO-TA will be able to set limits on strangelets of mass above $5 \times 10^{23} \mathrm{GeV} / \mathrm{c}^{2}$ that are more stringent than existing limits [23]. For this task a dedicated autonomous trigger is in preparation, as the external trigger from TAFD is designed to discard constant and slow moving events from the data stream such as airplanes.

The faintest meteor observed had an apparent magnitude of $M_{B}=2.4 \pm 0.11$, but was still very bright on the frames and easily detectable. If the same detection limit as for stars is assumed $-M_{B}=5.5$, then an average detection rate of about 1 meteor per hour of observation is expected, upon the introduction of a dedicated trigger algorithm.

A thunderstorm occurred during one of the observation campaigns of EUSO-TA, and the influence of lightning on the focal surface was registered. Due to the relatively slow timescale of lightning development relative to the packet length, without a dedicated trigger it was only possible to catch the rising slope of the phenomenon (Fig. 13). The thunderstorm took place outside of the field of view of the telescope ${ }^{3}$, thus no distinct features were visible. In separate tests it has been confirmed that the EUSO-TA electronics can be influenced by strong radio signals, resulting in rise of counts on the focal surface. In this way, it is not possible to conclude if the registered changes in counts are due to scattered light from the storm, or its radio emission.

High time resolution is required for observations of another thunderstorm related phenomena - TLEs - such as "sprites" and "elves", occurring high above the clouds on timescales of dozens of micro- to milliseconds. EUSO-TA should be able to detect some events of this kind after its planned upgrade (Section 5).

\subsection{Laser observations}

In order to study the response of EUSO-TA to a known light source the light coming from TA's CLF has been used, situated at a distance of about $21 \mathrm{~km}$ from EUSO-TA. The CLF shoots a laser of $355 \mathrm{~nm}$ wavelength vertically in front of the detectors [24].

During standard observations, the CLF is shot every half an hour for $30 \mathrm{~s}$ at a shooting frequency of $10 \mathrm{~Hz}$. The scattered light of

\footnotetext{
${ }^{3}$ Position of the thunderstorm with respect to the field of view was inspected visually by EUSO-TA shifters on-site.
}

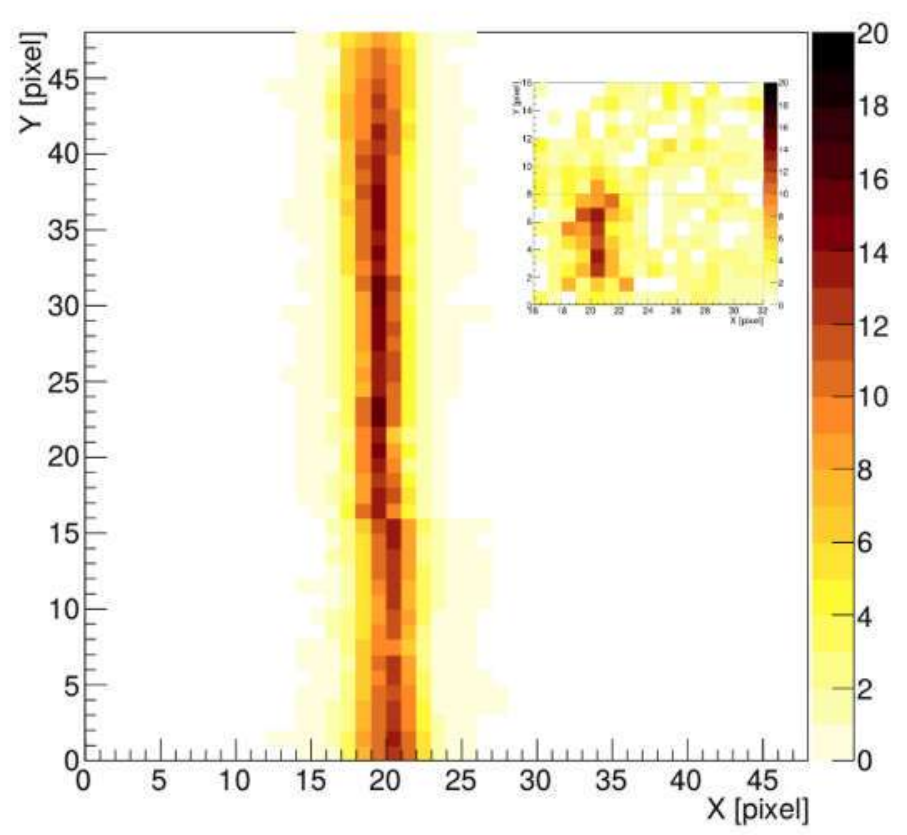

Fig. 14. An average of 259 tracks of the CLF laser, for a telescope elevation angle of $10^{\circ}$. The inset shows a part of a single frame containing the laser. The colour scale denotes the uncalibrated detector counts.

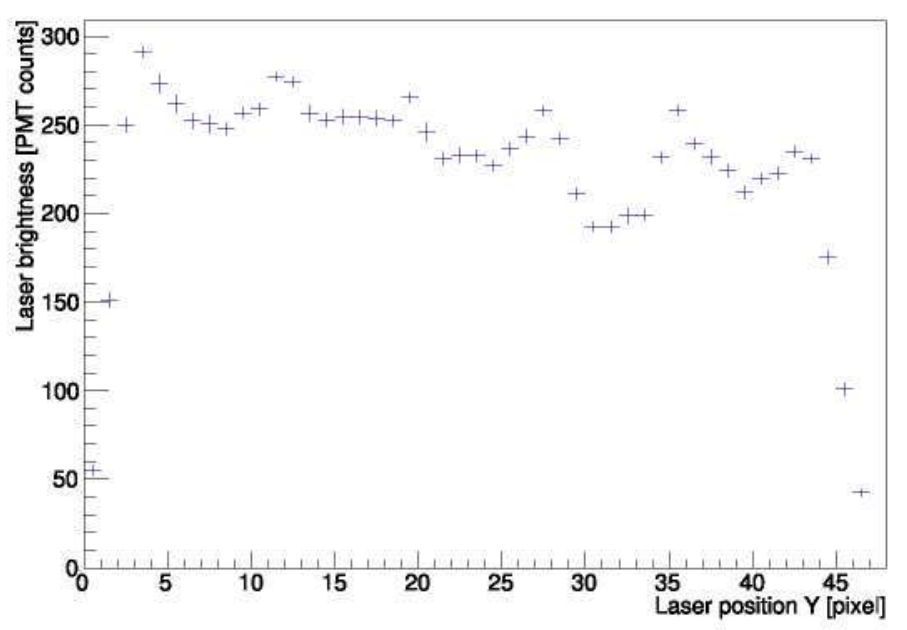

Fig. 15. A profile histogram of 259 CLF laser tracks, for telescope elevation angle of $10^{\circ}$. The values show the average of summed counts for laser spots in a specific position on the frame. The brightness is lower for spots falling into space between MAPMTs or outside the PDM.

the $\sim 3 \mathrm{~mJ}$ beam is clearly visible when traversing through the EUSO-TA field of view on 6 to 8 frames, depending on the shot and acquisition time synchronisation. The spot length is $6-8$ pixels depending on the position on the frame (Fig. 14, inset), which is consistent with expectations. The registered light intensity drops as the laser travels up in the field of view, as shown in Fig. 15. This is due to the increasing distance of the laser to the detector. The initial fast rise and final drop are due to the track entering and leaving the focal surface, while the intermediate drops are caused by dead spaces between MAPMTs. The brightness spread, defined as a standard deviation of the summed counts from a laser track in the EUSO-TA field of view, as measured by the detector is $5 \%$ (Fig. 16), comparable with an intrinsic CLF energy spread of $6 \%$.

In Fig. 17 the width of the CLF track as a function of its vertical position on the frame is shown, measured as a FWHM of a fitted gaussian profile. The width changes from about 3.8 pixels at the edges of the field of view to about 2.8 pixels in the centre of the 


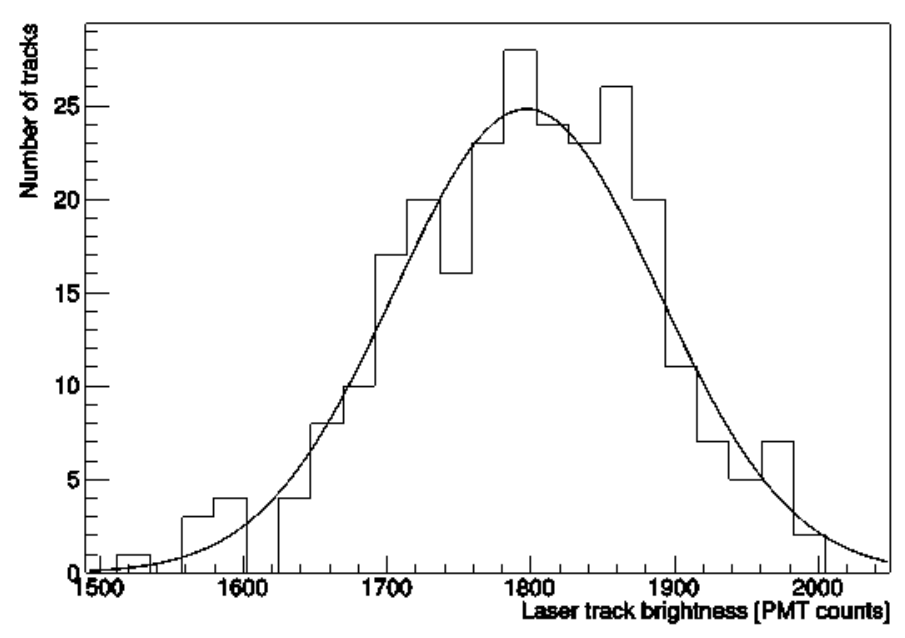

Fig. 16. The reconstructed distribution of the integrated number of counts from 259 CLF shots. The standard deviation of the fitted Gaussian function is about $5 \%$, comparable to the overall intrinsic CLF energy dispersion of $6 \mathrm{x}$.

frame. The last value is consistent with the measured PSF of star images. The dependency of the width on the position on the frame can be explained by the increasing influence of optical aberration with distance from the optical axis.

In addition to the CLF measurements, shooting of the Global Light System (GLS) [25] laser - a mobile UV laser of the Colorado School of Mines was also performed. The laser can be shot with energies in the range of about 1-86 m], with the pointing direction adjustable in two dimensions. The mechanics featured automatic changing of the pointing direction, allowing for easy "swipes" through the field of view. In addition the GLS laser produced events whose distance to the detector increased with time, as expected in space-based observation of cosmic rays. Initial results of the direction reconstruction can be seen in Fig. 18.

Fig. 19 shows reconstructed brightnesses of laser tracks in the detector vs the laser shot energy, for the distance of $33 \mathrm{~km}$. In the tested energy range of $4-22 \mathrm{~m}$, which is below the saturation of the detector, the dependency is linear, showing that EUSOTA behaves as expected. For the lowest energies of about $2-3 \mathrm{~mJ}$, only a few of the brightest laser events were reconstructed, resulting in a brightness cut on the data. While lowering the threshold of the reconstruction algorithm allowed for the detection of more events, the cut shows the point at which the detector starts to become limited by the background. The detection limit for the laser

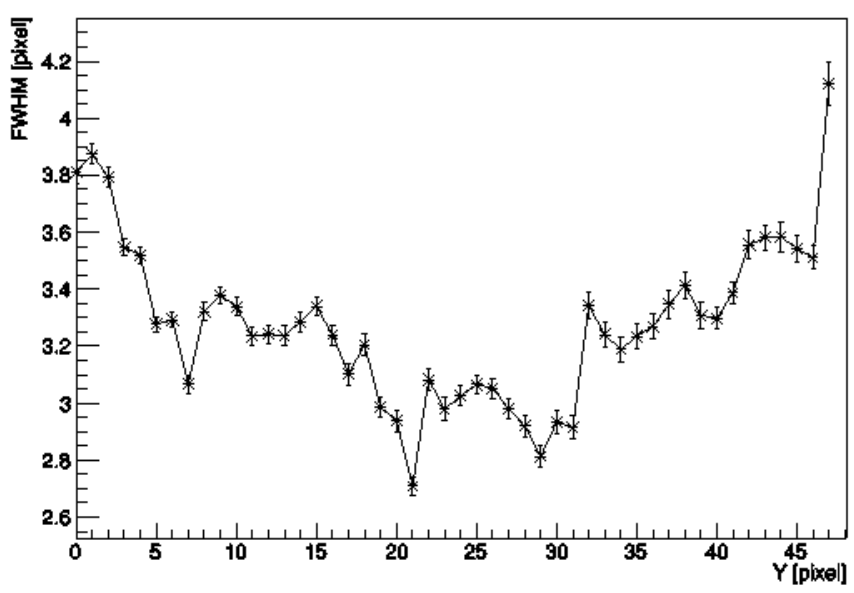

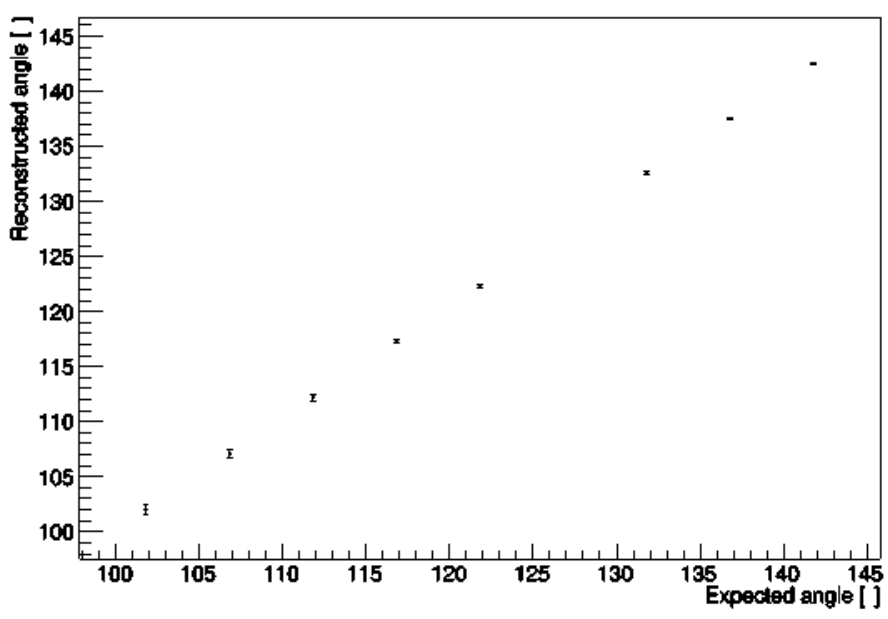

Fig. 18. Reconstructed angle vs expected angle for CiLS Iaser pointing sweep. Barely visible error bars show the standard deviation of the distribution of reconstructed angles.

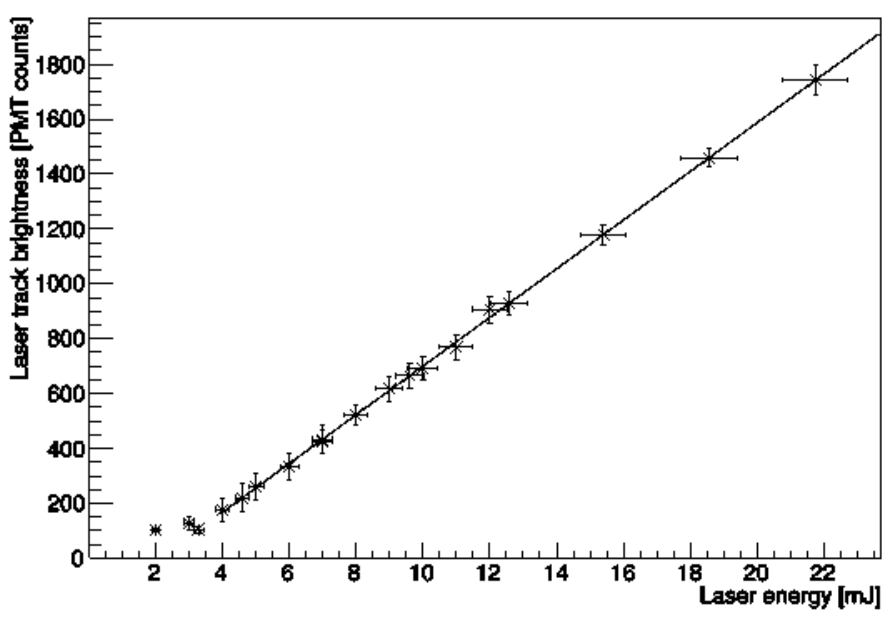

Fig. 19. Summed signal of the GLS laser track vs its energy for the distance of $33 \mathrm{~km}$ from the detector. The plot sums 2 shooting sessions, altogether encompassing an energy range of 2-22 mJ. Each point was calculated from a few dozens shots. Fitted line shows good linearity of the detector response in the range of 4-22 $\mathrm{m}$, while for lower energies only the brightest tracks were teconstructed, enforcing a low brightness cut on the data points.

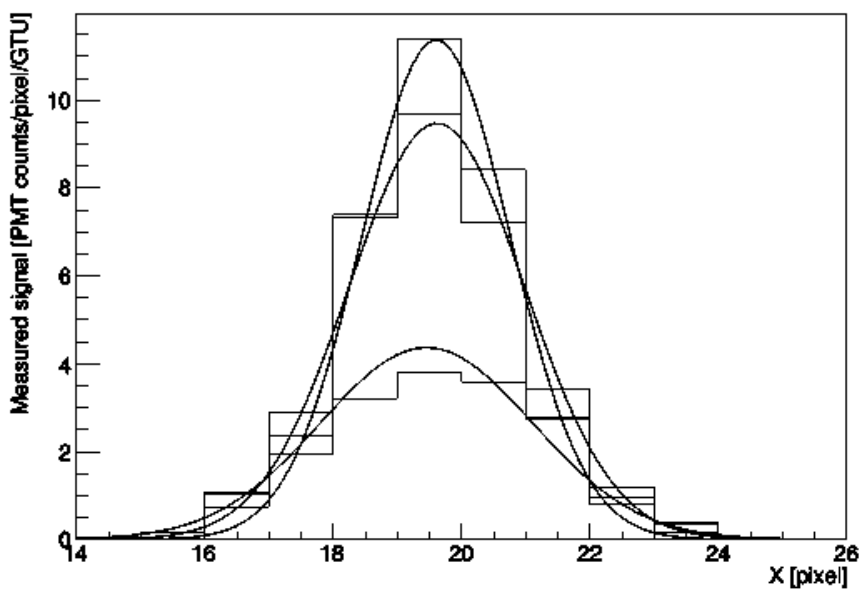

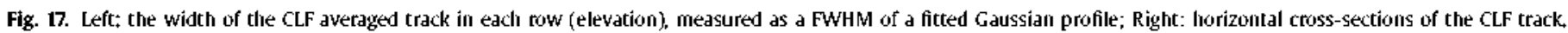
Three histograms show cross-sections in $Y$ positions cortesponding to the largest, smallest and intermediate widtlis, with fitted Gaussian profiles. 

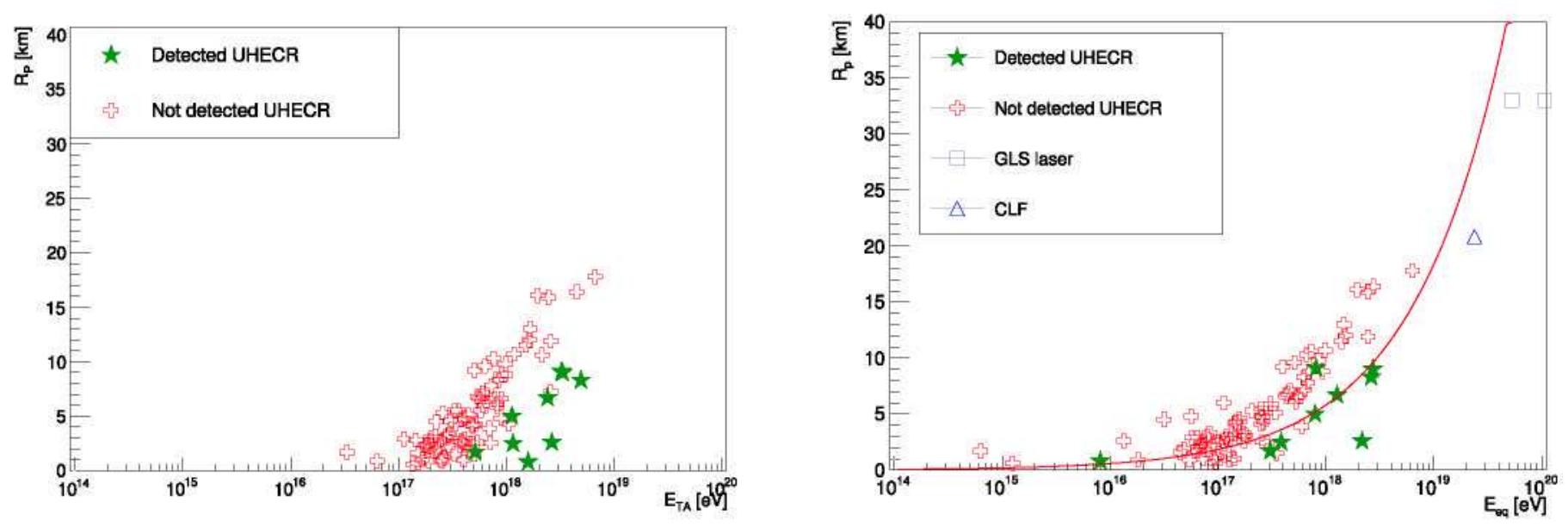

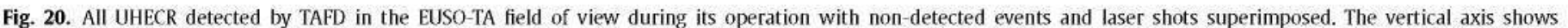

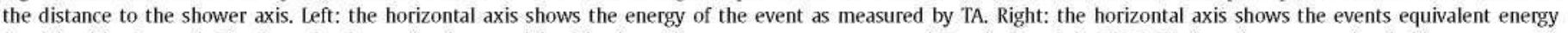
(explained in the text). The fit to the detected points, explained in the main text, suggests a conservative estimate of the EUSO-TA detection energy threshold.

shots of energies 2-4 mJ implies an energy threshold for detectable UHECR of energies $10^{19.7}-10^{20} \mathrm{eV}[26]$ at $33 \mathrm{~km}$ distance. However, it was also possible to detect a few shots of $1 \mathrm{~mJ}$ energy shot vertically from $33 \mathrm{~km}$ distance in the EUSO-TA data.

\subsection{UHECR}

To date, 9 UHECR events (Fig. 20) have been identified in 130 hours of UHECR-dedicated observations. The distances of these events from the detector vary between approximately 1 and $9 \mathrm{~km}$, while the energy is between $10^{17.7}-10^{18.8} \mathrm{eV}$, according to the TAFD reconstruction. The proximity of the events and the dead time between frames makes 8 events visible in the detector for a duration of a single frame, and one event for two frames. EUSOTA does not usually observe the maximum of the shower, but a late stage of the shower development, and as such the number of registered photons corresponds to a shower of lower energy than if the instrument was optimally pointed towards the shower maximum. Therefore, to estimate the instrument's capabilities it was necessary to calculate the equivalent energies of the events $\left(E_{e q}\right)$, corresponding to the reconstructed energy assuming that EUSO-TA observed the event's shower maximum. This calculation is based on the parameters measured by TA for each individual shower. The corresponding points can be used to form a conservative estimate of the detector's energy threshold. In a very simplified approach, one can assume that the minimal number of counts on the focal surface for the cosmic ray to be detected is constant, proportional to its energy and reversely proportional to the square of the distance from the shower axis $\left(R_{p}\right)$. Based on this assumption, $\mathrm{R}_{\mathrm{p}}=A \cdot \sqrt{E_{\text {eq }}}$ is fit to detected events, where $\mathrm{A}$ is a free parameter. It can be seen that the strong signals of the CLF shots (EAS equivalent energy of $\sim 10^{19.4} \mathrm{eV}$ at a distance of $21 \mathrm{~km}$ ) and GLS laser shots (EAS equivalent energy of $\sim 10^{19.7} \mathrm{eV}$ and $\sim 10^{20} \mathrm{eV}$ at a distance of $33 \mathrm{~km}$ ) are on the right side of the curve, i.e. in the detectable region, as expected.

Two typical events are shown in Fig. 21. It can be seen that rebinning of the images significantly increases the visibility of the tracks - making EUSO-TA data more similar to those of groundbased UHECR telescopes, which have much larger pixel sizes. However, such a pixel size is not suited for space-based observations, to which EUSO-TA has been tuned. Simulations of the events made with the "OffLine" package [27] are also presented here. The shower image can be reproduced to very fine detail, however, some overestimation of the signal in the simulation can be spotted on the residuals shown in Fig. 22 (left). Positive residuals, shown in the picture, align mainly in the shower area, while negative, which were excluded here for the sake of clarity, are randomly scattered over the whole field of view. Therefore the higher UHECR counts in the simulation cannot be attributed solely to statistical fluctuations. This difference can come both from intrinsic characteristics of the model and from uncertainties of shower parameters as measured by TA. As this problem will be addressed in the future development of the Offline package, the detailed analysis of residuals is not presented in this paper.

The small pixel size of EUSO-TA decreases the sensitivity but in turn gives higher spatial resolution compared to most other fluorescence telescopes. This allows for the study of the transversal profile of an EAS, as shown on Fig. 22 (right). The presented shower has a FWHM of 5.27 pixels.

In principle EUSO-TA should see only the fraction of UHECR detected by TAFD which both cross the field of view and are above the energy threshold. However, with the implementation of the external trigger, data is collected for each TAFD event. Therefore, an event is considered as detected if a linear trace is found in the EUSO-TA data and a corresponding event in TAFD results.

Simulations studies carried out in 2011 [13], prior to the installation of the telescope, predicted the detection of about 15 events in the total data acquisition time achieved to date. However, that analysis assumed more optimal EUSO-TA elevation angle of $30^{\circ}$, instead of the most often used $10^{\circ}-15^{\circ}$. Additionally, the analysis assumed a lower background light level than what was measured. In 2016, further simulations were performed, using the updated detector parameters [28], resulting in 8 predicted events, consistent with the 9 UHECR observed to date.

These first UHECR events registered with EUSO technology allowed for an important improvement and optimisation of the reconstruction and simulation software. However, the EAS parameters had to be derived from TAFD which, thanks to its larger field of view and higher time-resolution, could see the full shower development. Investigation of the parameters of the UHECR events which occurred inside the EUSO-TA field of view during its operation, starts to reveal the detection capabilities of the detector.

\section{EUSO-TA future plans}

EUSO-TA provides an excellent opportunity to test technology for existing and future experiments within the EUSO framework, as it allows for stable field observations for extended time periods. However, the observation time could be significantly increased if 
Data
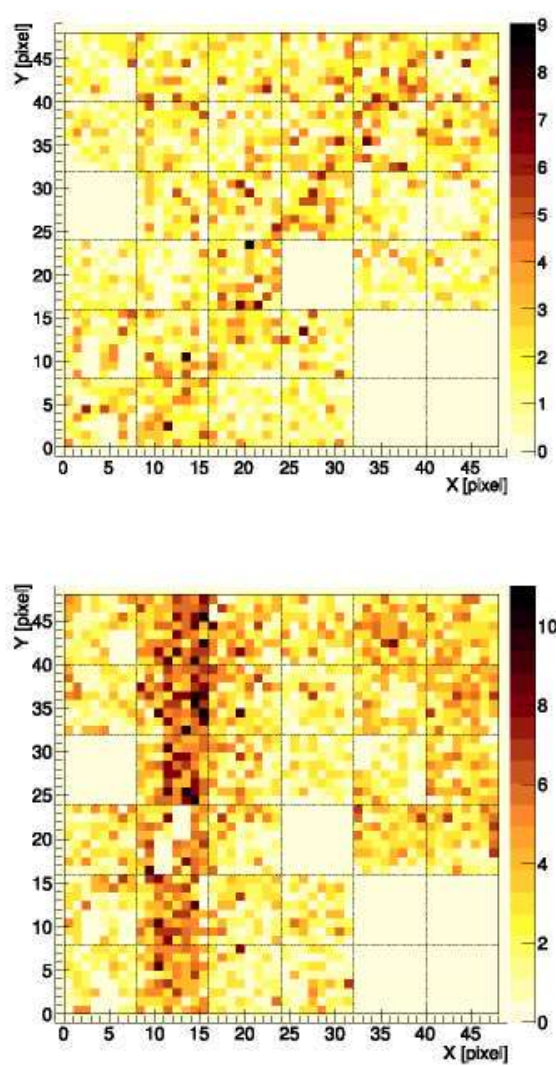

Data rebinned $2 \times 2$
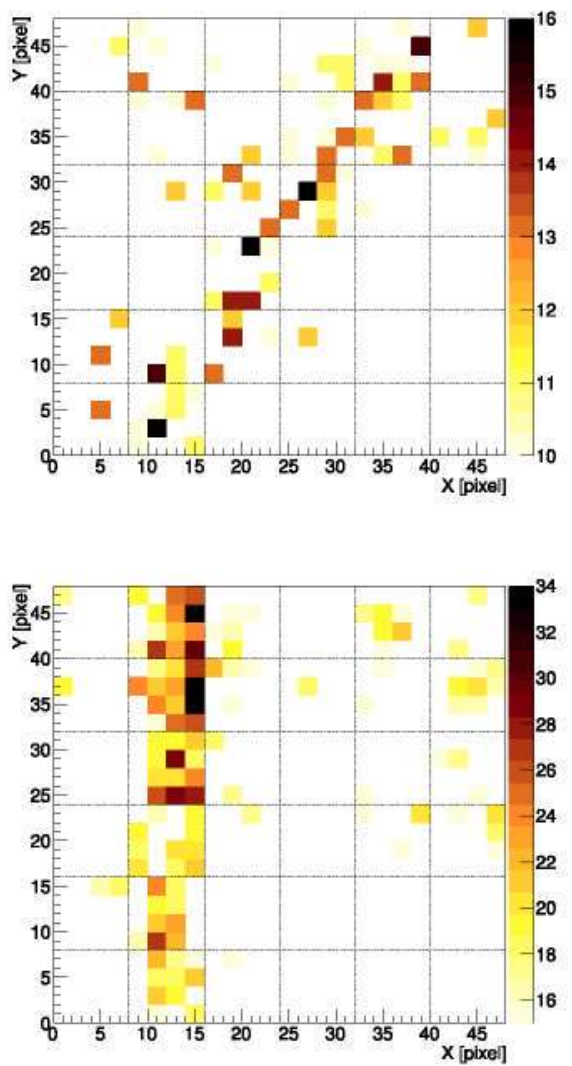

Simulation
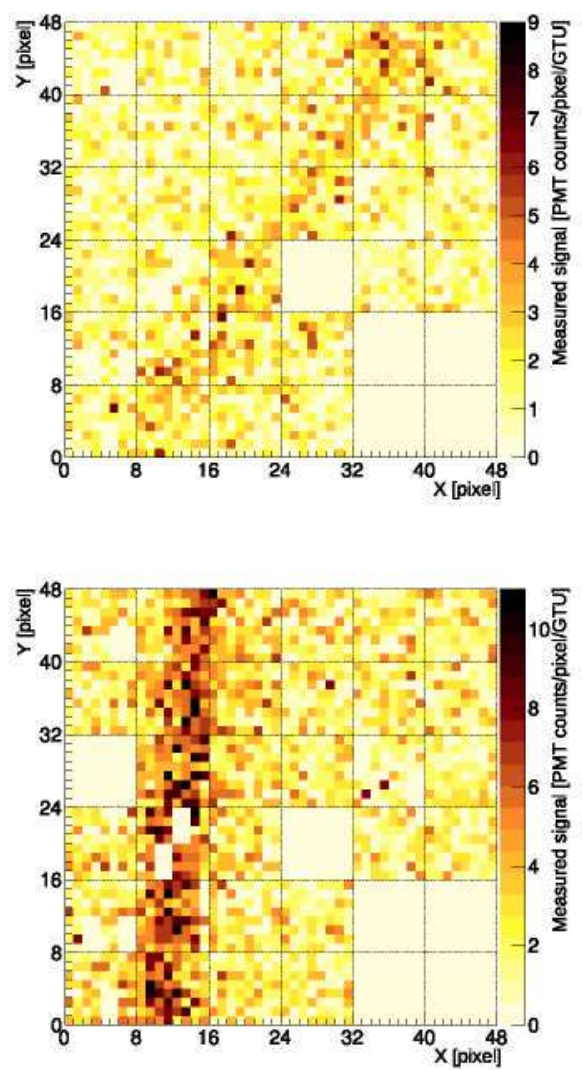

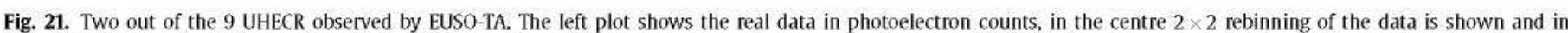

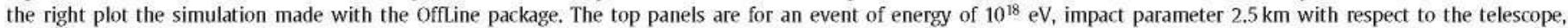

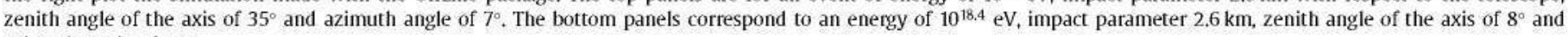
azimuth angle of $82^{\circ}$.
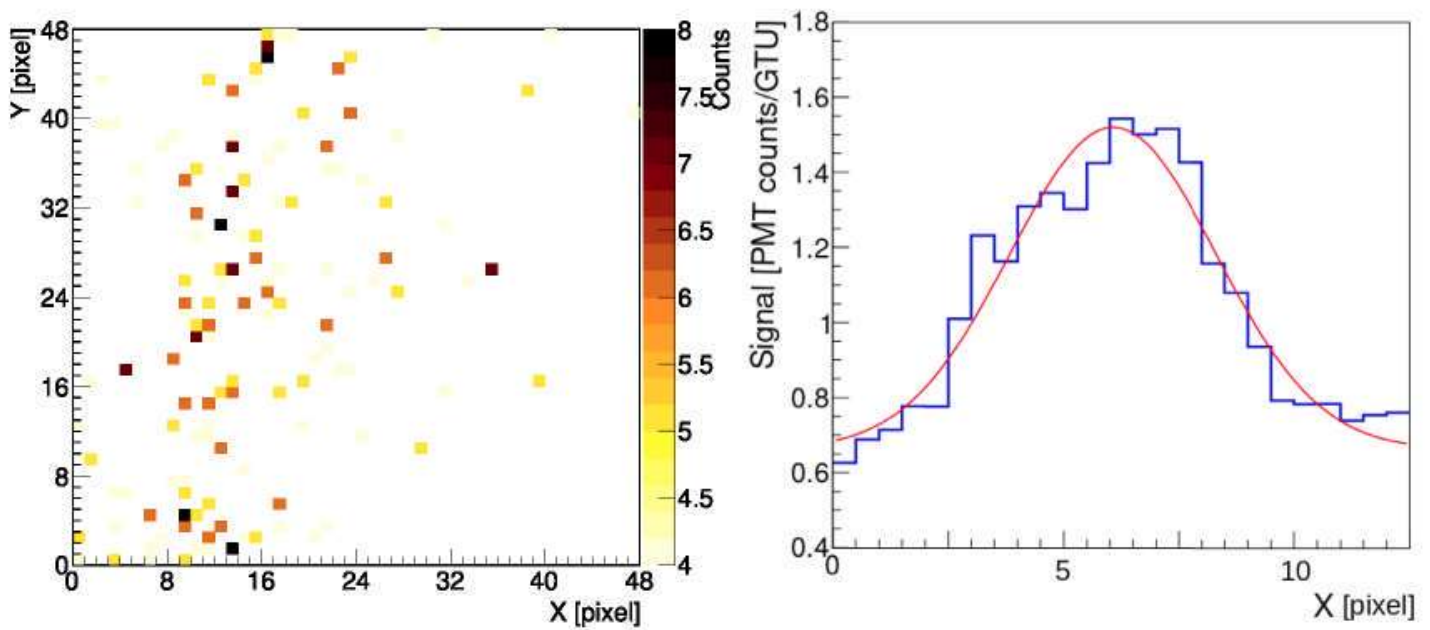

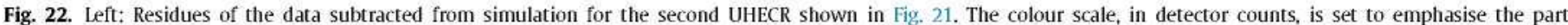

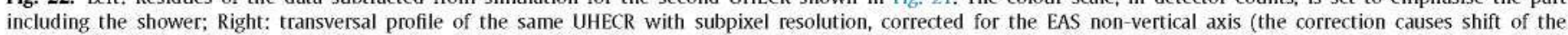
maximum compared to the original shower axis and X axis crossing point). The Gaussian fit gives FWHM of 5.27 pixels.

remote automatic operation of the instrument was implemented. This would allow for the collection of data continuously over the year in all possible observational periods. This task will require introduction of some additional mechanical and control devices, as well as a non-remote automatic working test period.

The sensitivity of the experiment will be enhanced with an upgrade of the EC-ASIC boards to the new version incorporating SPACIROC3 ASIC, which has $\sim 5$ ns single pulse resolution instead of the current $30 \mathrm{~ns}$. This parameter is important for nearby showers, which cross a single pixel in short timescales. Higher single pulse resolution decreases a chance of several photons from such an event falling into a single time bin and therefore being lost. The tests with EUSO-SPB in Utah show about a factor of 2 higher signal for CLF tracks, which may be attributed mainly to the use of SPACIROC3. The efficiency change should be much higher for close tracks, where the time spent in a single pixel is much shorter. 
The experiment will also be upgraded with advanced selftriggering capabilities. This will be achieved by replacing the current PDM data processing board with a new board based on system-on chip - Zynq XC7Z030 FPGA, recently developed by XILINX, which has more memory and resources. It allows the implementation of data read-out on three timescales, similar to the readout designed for the Mini-EUSO mission. This $\mu$ s timescale readout is self-triggered, dedicated to UHECR observations. Integrated packets forming 320 ms frames would be passed to second level trigger, dedicated mainly to atmospheric phenomena. Finally, ms scale frames integrated to $5.24 \mathrm{~s}$ exposures would be stored as a "movie" for analysis of slow phenomena, such as strangelets and meteors, detectable with offline event search algorithms.

\section{Conclusion}

EUSO-TA demonstrates the performance of a new technology for the observation of cosmic rays, based on using Fresnel lenses and multi-anode photomultipliers. The detector has registered, using TAFD triggers, 9 UHECR during its five observational campaigns, proving that use of Fresnel lenses and multi-anode photomultipliers works well for this purpose. The response of the detector was tested using UV laser shots mimicking extensive air showers. Additionally, a number of "slow" events such as stars, meteors and airplanes has been observed allowing for an extension of the scientific objectives.

The main goal of EUSO-TA, was to test the capabilities and stability of the hardware. It proved an invaluable testbench for the modifications applied in the EUSO-SPB and Mini-EUSO detectors.

From the 9 registered UHECR an idea of EUSO-TA sensitivity starts to emerge, which is found to be within expectations, especially for one of the first prototypes employing a new technology. Moreover, if the exposure time was reduced from the value of $2.5 \mu \mathrm{s}$ optimised for space-based measurements to the $100 \mathrm{~ns}$ used in TAFD as optimised for ground-based measurements, the signal to noise ratio would increase significantly. A similar effect will be achieved through the application of planned hardware upgrades in the future.

Results described in this article allowed us to evaluate the performance of an optical system composed of two Fresnel lenses, which behaved according to predictions. Fresnel-based optics have the advantage of wide field of view, simple design and reduced weight compared to mirror based systems. However, the disadvantages include the increased point spread function (compared to mirrors), chromatic aberration (which can be offset by diffractive lenses) and lower optical transmission due to several refractions. The advantages of one design over another in space-based systems depends strongly on the mission profile (rocket accommodation, satellite or space station, etc.).

A challenging future goal is keeping EUSO-TA up to date with developments of the other experiments in the EUSO framework. Two detectors - the already launched EUSO-SPB and the prepared Mini-EUSO - use a new version of EC-ASIC boards equipped with SPACIROC 3 ASICs, resulting in significantly higher dynamic range and signal to noise ratio. Mini-EUSO also replaces a PDM, CCB and Clock boards with the PDM data processing board allowing for the efficient and parallelised performance of more computationally demanding tasks. Introducing these elements to EUSO-TA is a necessary step to increase its performance and keep compatibility with other missions. In the near future an upgrade and automatisation of the telescope is planned to increase its sensitivity and duty cycle. Also, it is hoped to include an additional middle diffractive Fresnel lens to reduce the PSF and thus increase the signal to noise ratio. Finally, EUSO-TA will be used as a testbench for the development of multi-PDM observations and readout.

\section{Acknowledgments}

This work was partially supported by Basic Science Interdisciplinary Research Projects of RIKEN and JSPS KAKENHI Grant (22340063, 23340081, and 24244042), by the Italian Ministry of Foreign Affairs and International Cooperation, by the Italian Space Agency through the ASI INFN agreement no, 2017-8-H.0, by NASA award 11-APRA-0058 in the USA, by the Deutsches Zentrum für Luft- und Raumfahrt, by the French space agency CNES, the Helmholtz Alliance for Astroparticle Physics funded by the Initiative and Networking Fund of the Helmholtz Association (Germany), by Slovak Academy of Sciences MVTS JEM-EUSO, by National Science Centre in Poland grant (2015/19/N/ST9/03708), by Mexican funding agencies PAPIT-UNAM, CONACYT and the Mexican Space Agency (AEM), as well as VEGA grant agency project 2/0132/17. and by State Space Corporation ROSCOSMOS and Russian Foundation for Basic Research (grant 16-29-13065). We are grateful to the Telescope Array collaboration for allowing us to use the TA site and its facilities, and for all their help.

We dedicate this article to Yoshiya Kawasaki and Jacek Karczmarczyk, who passed away in 2016.

\section{References}

[1] Y. Takahashi, J.-E. Collaboration, et al., The JEM-EUSO mission, New J. Phys. 11 (6) (2009) 065009.

[2] L. Porter, J. Earnshaw, E. Tielsch-Cassel, J. Ahlstrom, K. Greisen, A space-time detector for cosmic ray showers, Nucl, Instrum. Methods 87 (1) (1970) 87-92

[3] J. Adams, S. Ahmad, ].-N. Albert, D. Allard, L. Anchordoqui, V. Andreev, A. Anzalone, Y. Arai, K. Asano, M.A. Pernas, et al., The EUSO-Balloon pathfinder, Exp. Astron. 40 (1) (2015) 281-299.

[4] M. Ricci, M. Casolino, P. Klimov, Mini-EUSO: a pathfinder for JEM-EUSO to measure earths UV background from the ISS, Proc. Science. PoS (ICRC2015) 599 (2015).

[5] B.A. Khrenov, V.V. Alexandrov, D.I. Bugrov, G.K. Garipov, N.N. Kalmykov, M.I. Panasyuk, S.A. Sharakin, A.A. Silaev, I.V. Yashin, V.M. Grebenyuk, D.V. Naumov, A.G. Olshevsky, B.M. Sabirov, R.N. Semenov, M. Slunechka, I.I. Skryl, L.G. Tkatchev, O.A. Saprykin, V.S. Syromyatnikov, V.E. Bitkin, S.A. Eremin, A.I. Matyushkin, F.F. Urmantsev, V. Abrashin, V. Koval, Y. Arakcheev, A. Cordero, O. Martinez, E. Morena, C. Robledo, H. Salazar, L. Villasenor, A. Zepeda, I. Park, M. Shonsky, J. Zicha, KLYPVE/TUS Space experiments for study of ultrahighenergy cosmic rays, Phys. At. Nucl. 67 (11) (2004) 2058-2061, doi:10.1134/1. 1825529.

[6] P. Klimov, C. M., Status of the KLYPVE-EUSO detector for EECR study on board the ISS, Proc. ICRC2017 (2017).

[7] Y. Tameda, et al,, Telescope array experiment, Nuclear Phys, B 196 (2009) $74-79$.

[8] H. Prieto-Alfonso, L. del Peral, M. Casolino, K. Tsuno, T. Ebisuzaki, M. Frías, J.-E, Collaboration, Multi anode photomultiplier tube reliability assessment for the JEM-EUSO space mission, arXiv:1501.05908 (2015).

[9] H. Miyamoto, K. Yoshida, F. Kajino, S. Ahmad, P. Barrillon, S. Blin-Bondil, S. Dagoret-Campagne, C. de la Taille, F. Dulucq, P. Gorodetzky, et al., Performance of the SPACIROC front-end ASIC for JEM-EUSO, JEM-EUSO Mission Contrib. ICRC 2013 (2013) 103.

[10] J. Bayer, G. Distratis, D. Gottschall, A. Santangelo, C. Tenzer, M. Bertaina, M. Casolino, G. Osteria, J.-E. Collaboration, Second level trigger and Cluster control Board for the JEM-EUSO mission, in: Proceedings of 33rd International Cosmic Ray Conference, Rio de Janeiro, Brazil, 2013, pp. 99-102.

[11] F. Kajino, M. Casolino, T. Ebisuzaki, J. Adams, P. Ballmoos, M. Bertaina, M. Christl, S. Dagoret, C. De La Taille, M. Fukushima, et al., The JEM-EUSO Instruments, in: 33rd International Cosmic Ray Conference (ICRC2013), 2013.

[12] V. Scotti, G. Osteria, on behalf of the JEM-EUSO Collaboration, The Data Processor of the JEM-EUSO pathfinders, in: Astroparticle, Particle, Space Physics and Detectors for Physics Applications, pp. 120-124.

[13] L.W. Piotrowski, M. Casolino, L. Conti, T. Ebisuzaki, C. Fornaro, Y. Kawasaki, Y. Hachisu, H. Ohmori, C. De Santis, K. Shinozaki, et al., On-line and off-line data analysis for the EUSO-TA experiment, Nucl. Instrum. Methods Phys. Res., Sect. A 773 (2015) 164-171.

[14] T. Fujii, M. Malacari, M. Bertaina, M. Casolino, B. Dawson, P. Horvath M. Hrabovsky, J. Jiang, D. Mandat, A. Matalon, et al., Detection of ultra-high energy cosmic ray showers with a single-pixel fluorescence telescope, Astropart. Phys. 74 (2016) 64-72.

[15] ]. Adams, E. Kuznetsov, J. Watts, S. Csorna, M. Rodencal, LW. Piotrowski, M. Mustafa, Calibration of the EUSO-TA prototype instrument, PoS (2015) 582

[16] H. Johnson, W. Morgan, Fundamental stellar photometry for standards of spectral type on the revised system of the yerkes spectral atlas, Astrophys. J. 117 (1953) 313. 
[17] M.A. Perryman, L. Lindegren, J. Kovalevsky, E. Hoeg, U. Bastian, P. Bernacca, M. Crézé, F. Donati, M. Grenon, M. Grewing, et al., The HIPPARCoS catalogue, Astron. Astrophys. 323 (1997) L49-L52.

[18] L. Piotrowski, T. Batsch, H. Czyrkowski, M. Cwiok, R. Dabrowski, G. Kasprowicz, A. Majcher, A. Majczyna, K. Malek, L. Mankiewicz, et al., PSF Modelling for very wide-field CCD astronomy, Astron. Astrophys. 551 (2013) A119.

[19] Z. Plebaniak, J. Szabelski, T. Wibig, L. Piotrowski, et al., Point Spread Function of EUSO-TA detector, in: Proceedings of 35th International Cosmic Ray Conference, Busan.

[20] J. Abraham, P. Abreu, M. Aglietta, C Aguirre. E. Ahn, D. Allard, I. Allekotte J. Allen, P. Allison, J. Alvarez-Muniz, et al., The fluorescence detector of the pierre auger observatory, Nucl. Instrum. Methods Phys. Res., Sect. A 620 (2) (2010) 227-251.

[21] M. Hayashi, Y. Tameda, T. Tomida, Y. Tsunesada, T. Seki, Y. Saito, Development of a UAV-mounted Light Source for Fluorescence Detector Calibration of the Telescope Array Experiment, in: Proceedings of 2016 International Conference on Ultra-High Energy Cosmic Rays (UHECR2016), 2018, p. 011036.

[22] T. Ebisuzaki, M.N. Quinn, S. Wada, L.W. Piotrowski, Y. Takizawa, M. Casolino, M.E. Bertaina, P. Gorodetzky, E. Parizot, T. Tajima, et al., Demonstration designs for the remediation of space debris from the international space station. Acta Astronaut. 112 (2015) 102-113.
[23] J. Adams, S. Ahmad, J--N. Albert, D. Allard, L. Anchordoqui, V. Andreev, A. Anzalone, Y. Arai, K. Asano, M.A. Pernas, et al., JEM-EUSO: Meteor and nuclearite observations, Exp. Astron. 40 (1) (2015) 253-279.

[24] Y. Takahashi, K. Yamazaki, D. Ikeda, H. Sagawa, H, Tokuno, K. Hayashi M. Fukushima, S. Ogio, S. Udo, T. Fujii, et al., Central laser facility analysis at the Telescope Array experiment, in: AIP Conference Proceedings, vol. 1367, AIP, 2011, pp. 157-160,

[25] P. Hunt, R. Bachman, J. Eser, M. Mantz, L. Wiencke, C. Baron, C. Bigler, D. Bruzgo, M. Burg, A. Cain, A. Cummings, A. Evans, W. Finch, J. Fruit, T. Gallmeyer, C. Geier, J. Gossman, D. Hirsch, R. Hanley, A. Osieczanek, I, Smith, T. Wills, for the JEM-EUSO Collaboration, The JEM-EUSO global light system laser station prototype, PoS (ICRC2015) 236 (2016).

[26] A.L. Cummings, Field Testing for EUSO-SPB: Logistics and First Results, Ph.D. thesis, Colorado School of Mines. Arthur Lakes Library,

[27] T. Paul, M. Bertaina, F. Bisconti, J. Eser, G. Figueiredo, M. Fouka, F. Guarino, L Del Peral, Z. Sahnoun, et al., New simulation and reconstruction software for the EUSO pathfinders, with example applications, Proc, ICRC2017 (2017).

[28] F. Bisconti, et al., Simulation study of the detected and expected events for the EUSO-TA fluorescence detector, in: Proceedings of 35th International Cosmic Ray Conference, Busan, Korea, 2017. 